\title{
Operator Algebra of Foliations with Projectively Invariant Transverse Measure
}

\author{
by \\ Makoto YAMASHITA
}

\begin{abstract}
We study the structure of operator algebras associated with the foliations which have projectively invariant measures. When a certain ergodicity condition on the measure preserving holonomies holds, the lack of holonomy invariant transverse measure can be established in terms of a cyclic cohomology class associated with the transverse fundamental cocycle and the modular automorphism group.
\end{abstract}

2010 Mathematics Subject Classification: Primary 58B34; Secondary 46L87.

Keywords: noncommutative geometry, foliation, von Neumann algebra, cyclic cohomology

\section{$\S 1$. Introduction}

In [C4], A. Connes introduced the von Neumann algebra $W(M ; F)$ associated with a foliation $F$ on a manifold $M$. He showed that several measure-theoretic properties of the space of leaves $M / F$ can be stated in terms of $W(M ; F)$. For example, the ergodic components of $M / F$ correspond to the continuous decomposition of $W(M ; F)$, and the lack of holonomy invariant measure in the absolute continuity class of transverse Lebesgue measure is equivalent to $W(M ; F)$ being a type III algebra. Subsequently it was shown by S. Hurder and A. Katok [HK] and Connes [C7] that if a foliation $F$ admits a nonzero generalized Godbillon-Vey class $\omega$, then the direct summand of $W(M ; F)$ corresponding to the support of $\omega$ is of type III.

When one considers the holonomy of transverse coordinates, there is an interesting class of foliations with simplified transverse structure, namely that of transversely affine foliations. For these, several sufficient conditions for the existence of an invariant transverse measure were established by W. M. Goldman, M.

Communicated by H. Okamoto. Received November 22, 2010. Revised May 7, 2012, and February $18,2013$.

M. Yamashita: Graduate School of Mathematical Sciences, the University of Tokyo; e-mail: makotoy@ms.u-tokyo.ac.jp 
W. Hirsch, and G. Levitt [GHL]. There are also studies of more specific examples in this class, for instance the ones given by hyperbolic automorphisms of tori, by J. F. Plante $[\mathrm{P}]$.

In this paper we investigate the class of foliations with positive projectively invariant measures, naturally including the transversely affine foliations. Our main theorem gives a sufficient condition (Theorem 2) for $W(M ; F)$ to be of type III in terms of a certain cyclic cocycle $i_{D} \phi$ and its pairing with the $K$-group of the foliation algebra. Although we closely follow Connes's method to establish the lack of invariant transverse measure, we note that the Godbillon-Vey classes themselves can be trivial in our setting (Remark 15). We also obtain a more precise description (Theorem 3) of the pairing of $i_{D} \phi$ with $K_{*}\left(C_{r}^{*}(M ; F)\right)$ when $W(M ; F)$ is of type $\mathrm{III}_{\lambda}$ for $0<\lambda<1$.

This paper is organized as follows. Section 2 is devoted to a review of basic groupoid and algebra constructions associated with foliations. Next in Section 3, we give a description (Corollary 11) of the image of invariant cyclic cocycles under the 'boundary map' in R. Nest's Pimsner-Voiculescu type exact sequence [N] of periodic cyclic cohomology. In Section 4, we show that the transverse fundamental cocycle $\phi$ is invariant under the modular automorphism group if the restricted holonomy groupoid admits a projective invariant density. Due to this invariance, one obtains a cyclic cocycle of degree $q+1$ over the smooth convolution algebra of $F$ as the 'interior product' $i_{D} \phi$ of the generator of the modular automorphism group with $\phi$. This cocycle is anabelian in the sense of Connes, and its pairing with a class in the $K$-group gives rise to an invariant measure on the flow of weights, leading to the theorems mentioned above.

\section{$\S 2$. Preliminaries}

\section{$\S 2.1$. Basic definitions of foliation algebras}

Let $M$ be a smooth oriented manifold of dimension $n$, and $F$ a smooth oriented foliation of dimension $p$ on $M$. We let T $M$ denote the tangent bundle of $M$ and identify $F$ with an integrable subbundle of T $M$. The normal bundle TM/F of $F$ is denoted by $\tau$, and the codimension $n-p$ of $F$ is by $q$. Thus $\wedge^{q} \tau^{*}$ can be regarded as the bundle of signed transverse densities. Let $\operatorname{Det}_{\tau}^{+}(M)$ denote the bundle of nonzero transverse densities over $M$, which is a subbundle of $\bigwedge^{q} \tau^{*}$ determined by the orientation on $F$. It is a principal $\mathbb{R}_{>0}$-bundle over $M$.

Let $G$ be the holonomy groupoid of $F$. We let $r, s: G \rightarrow M$ denote the range and source maps of $G$. When $x$ is a point of $M$, the set of holonomies with source $x$ is denoted by $G_{x}$. When $x$ and $y$ are points on the same leaf of $F$, we put $G_{x}^{y}=$ 
$\{\gamma \in G \mid s(\gamma)=x, r(\gamma)=y\}$. The composition of holonomies is written as

$$
G_{y}^{z} \times G_{x}^{y} \rightarrow G_{x}^{z}, \quad\left(\gamma, \gamma^{\prime}\right) \mapsto \gamma \gamma^{\prime},
$$

for any triple $x, y, z$ of points of $M$ on the same leaf. When $T \subset M$ is a transversal (of dimension $q$ ) of $F$, the restricted groupoid $G_{T}=\{\gamma \in G \mid r \gamma, s \gamma \in T\}$ becomes an étale groupoid over $T$.

Even though $G_{T}$ might not be a Hausdorff space, it is possible to take a covering of $G_{T}$ by open sets that are homeomorphic to $\mathbb{R}^{q}$. A function on $G_{T}$ is said to be compactly supported smooth if it is so when restricted to one such open set. The space of compactly supported smooth functions is denoted by $C_{c}^{\infty}\left(G_{T}\right)$. As in [C5, Section 6], the formula $f * f^{\prime}(\gamma)=\sum_{\gamma=\gamma^{\prime} \gamma^{\prime \prime}} f\left(\gamma^{\prime}\right) f^{\prime}\left(\gamma^{\prime \prime}\right)$ defines an associative algebra structure on $C_{c}^{\infty}\left(G_{T}\right)$.

There is a naturally induced action of the groupoid $G$ on the bundle $\tau$ over $M$. We make an identification of the tangent bundle TT of $T$ with $\left.\tau\right|_{T}$ given by the composition of the inclusion $\left.\mathrm{T} T \rightarrow \mathrm{T} M\right|_{T}$ and the projection $\left.\left.\mathrm{T} M\right|_{T} \rightarrow \tau\right|_{T}$. The action of $G$ on $\tau$ induces one of $G_{T}$ on TT via this identification.

A $G_{T}$-quasiinvariant measure on $T$ gives rise to a positive definite functional on the convolution algebra of $G_{T}$. In this paper we concentrate on measures of Lebesgue absolute continuity class among transverse densities. Hence they are given by sections of the $\mathbb{R}_{>0}$-bundle $\operatorname{Det}_{\tau}^{+}(T)$ which are Lebesgue measurable, and two such sections are identified when they agree off a negligible subset.

Given a foliation $F$ on $M$, a transversal $T$, and a density $\omega$ on $T$, one obtains an inner product on $C_{c}^{\infty}\left(G_{T}\right)$ by

$$
\left(f_{0}, f_{1}\right)=\int_{x \in T}\left(f_{0}, f_{1}\right)_{\ell^{2} G_{x}} \omega_{x}=\int_{x \in T} \sum_{\gamma \in G_{x}} f_{0}(\gamma) \overline{f_{1}(\gamma)} \omega_{x}
$$

Let $H$ denote the Hilbert space completion of $C_{c}^{\infty}\left(G_{T}\right)$ with respect to this inner product. Let us recall the definition of relevant operator algebras [C2, C5] on $H$ associated with the groupoid $G_{T}$.

Let $\mathcal{A}$ denote the *-algebra of functions in $C_{c}^{\infty}\left(G_{T}\right)$ endowed with the convolution product and the involution $f^{*}(\gamma)=\overline{f\left(\gamma^{-1}\right)}$. The left convolution

$$
\pi_{l}(f) \xi(\gamma)=\sum_{\gamma=\gamma^{\prime} \gamma^{\prime \prime}} f\left(\gamma^{\prime}\right) \xi\left(\gamma^{\prime \prime}\right)
$$

defines a *-representation of $\mathcal{A}$ on $H$. The operator norm closure $C_{r}^{*}\left(G_{T}\right)$ of $\mathcal{A}$ is called the reduced $C^{*}$-algebra of the foliation $(M ; F)$. The weak closure $W(M ; F)$ of $\pi_{l}(\mathcal{A})$ is called the von Neumann algebra of $(M ; F)$. These completions contain appropriate enlargements of $C_{c}^{\infty}\left(G_{T}\right)$ such as the space $C_{c}\left(G_{T}\right)$ of compactly sup- 
ported continuous functions, and we make use of these implicitly when there is no confusion.

For each leaf $l$ of $F$, let $\tilde{l}$ be the holonomy covering of $l$, and $\pi$ be the covering map $\tilde{l} \rightarrow l$. Thus, $\tilde{l}$ can be regarded as a copy of $G_{x}$ for any $x \in l$. Then $W(M ; F)$ can be described as the algebra of families $\left(T_{l}\right)_{l \in M / F}$ of operators indexed by the space of leaves of $F$ where $T_{l} \in B\left(\ell^{2}\left(\pi^{-1} T \cap \tilde{l}\right)\right)$, bounded in the sense that there exists a positive number $C$ satisfying $\left\|T_{l}\right\|<C$ for all $l$, and measurable in the sense that the function $\left(\gamma, \gamma^{\prime}\right) \mapsto\left(T_{l} e_{\gamma^{\prime}}, e_{\gamma \gamma^{\prime}}\right)$ is measurable on $G_{T} \times_{T} G_{T}$, and two presentations $\left(T_{l}\right)_{l \in M / F}$ and $\left(T_{l}^{\prime}\right)_{l \in M / F}$ are identified if they agree on a union of leaves whose complement has measure 0 .

The commutant $W\left(\nu_{\omega}\right)$ of $\pi_{l}(\mathcal{A})$ on $H$ is weakly generated by right convolution operators. Namely, there is an anti-representation of $\mathcal{A}$ on $H$ by

$$
\pi_{r}(f) \xi(\gamma)=\sum_{\gamma=\gamma^{\prime} \gamma^{\prime \prime}} \xi\left(\gamma^{\prime}\right) f\left(\gamma^{\prime \prime}\right)
$$

In contrast to the case of left convolution, this representation may not be a *-representation, and this failure can be measured by the modular function $\delta^{(\omega)}$ defined below. Nevertheless, operators of the form $\pi_{r}(f)$ for $f \in C_{0}\left(G_{T}\right)$ are bounded in the commutant of $\pi_{l}(\mathcal{A})$.

The center $Z(W(M ; F))=W(M ; F) \cap W\left(\nu_{\omega}\right)$ of $W(M ; F)$ is naturally identified with the algebra $L^{\infty}(T)^{G_{T}}$ of bounded measurable functions on $T$ which are invariant under the action of $G_{T}$. Hence $W(M ; F)$ is a factor if and only if the action of $G_{T}$ on $T$ is ergodic with respect to the Lebesgue measure class.

The density $\omega$ also gives a family $a_{l}$ of operator-valued densities [C4, C8]. When $X$ is a section of $\left.\bigwedge^{q} \tau\right|_{T}=\bigwedge^{q} \mathrm{~T} T$ and $l \in M / F$, the corresponding operator $a(X)_{l}$ on $H_{l}=\ell^{2}\left(\pi^{-1} T \cap \tilde{l}\right)$ is given by the diagonal operator $e_{x} \mapsto\left\langle\omega_{x}, X_{x}\right\rangle e_{x}$. It defines a weight $\phi^{(\omega)}$ on $W(M ; F)$ by the invariant integral

$$
\phi^{(\omega)}\left(\left(T_{l}\right)_{l \in M / F}\right)=\int_{T}\left(a_{l} T_{l} e_{x}, e_{x}\right)=\int_{T}\left(T_{l} e_{x}, e_{x}\right) \omega_{x}
$$

on $T$. The representation $\pi_{l}$ of $\mathcal{A}$ on $H$ can be identified with the GNS representation of $\phi^{(\omega)}$.

The modular function $\delta^{(\omega)}$ from $G_{T}$ to $\mathbb{R}_{>0}$ associated with $\omega$ is defined by

$$
\omega_{s \gamma}=\delta^{(\omega)}(\gamma) \gamma^{*} \omega_{r \gamma} .
$$

The density $\omega$ is invariant under holonomy transformations if and only if $\delta^{(\omega)}$ is identically equal to 1 on $G_{T}$.

Definition 1. The transverse density $\omega$ is said to be projectively invariant if the function $\delta^{(\omega)}$ is locally constant on $G_{T}$. 
Suppose that $\omega$ is projectively invariant, and let $\gamma \in G_{T}$ be represented by the germ of a local holonomy map $g: S \rightarrow g(S)$ defined on an open connected neighborhood $S$ of $s(\gamma)$. Then the set $U_{g}$ of holonomies defined by $g$ becomes an open connected neighborhood of $\gamma$ in $G_{T}$, and $\delta^{(\omega)}$ becomes a constant function on $U_{g}$. Thus, the pullback of $\left.\omega\right|_{g(S)}$ by $g$ is a scalar multiple of $\left.\omega\right|_{S}$ with the positive coefficient $\delta^{(\omega)}(\gamma)$.

Let $\sigma_{t}^{(\omega)}$ be the modular automorphism associated with the weight $\phi^{(\omega)}$. Thus, when $f$ is a function of compact support on $G_{T}$, its action on $\pi_{l}(f)$ is given by

$$
\sigma_{t}^{(\omega)}\left(\pi_{l}(f)\right)=\pi_{l}\left(\left(\delta^{(\omega)}\right)^{i t} f\right),
$$

where $\left(\delta^{(\omega)}\right)^{i t} f$ denotes the pointwise product of the functions $\delta^{(\omega)}(\gamma)^{i t}$ and $f(\gamma)$ on $G_{T}$.

Suppose that $\omega$ is smooth. Then $\delta^{(\omega)}$ is smooth on $G_{T}$, and (2) implies that the 1-parameter group $\sigma_{t}^{(\omega)}$ preserves the subalgebra $\pi_{l}\left(C_{c}^{\infty}\left(G_{T}\right)\right)$. When this is the case, we let $\mathbb{R} \ltimes_{\sigma^{(\omega)}} C_{c}^{\infty}\left(G_{T}\right)$ denote the linear space $\mathcal{S}\left(\mathbb{R} ; C_{c}^{\infty}\left(G_{T}\right)\right)$ of Schwartz class functions on $\mathbb{R}$ with values in $C_{c}^{\infty}\left(G_{T}\right)$, endowed with the convolution product twisted by the action $\sigma^{(\omega)}$.

Let $\operatorname{Det}_{\tau}^{+}(T)$ denote the restriction of $\operatorname{Det}_{\tau}^{+}(M)$ to $T$. By means of the section $\omega$ of $\operatorname{Det}_{\tau}^{+}(T)$, it can be identified with the direct product $\mathbb{R}_{>0} \times T$. The natural action of $G_{T}$ on $\operatorname{Det}_{\tau}^{+}(T)$ is identified with the action

$$
\gamma \cdot(r, x)=\left(\delta^{(\omega)}(\gamma) r, x\right)
$$

of $G_{T}$ on $\mathbb{R}_{>0} \times T$. We let $\operatorname{Det}_{\tau}^{+}\left(G_{T}\right)$ denote the corresponding groupoid $G_{T} \ltimes$ $\operatorname{Det}_{\tau}^{+}(T)$.

There is an injective homomorphism

$$
C_{c}^{\infty}\left(\operatorname{Det}_{\tau}^{+}\left(G_{T}\right)\right) \rightarrow \mathbb{R} \ltimes_{\sigma^{(\omega)}} C_{c}^{\infty}\left(G_{T}\right)
$$

with dense image, given by the Fourier transform on the $\mathbb{R}$-coordinate in $\mathbb{R} \times T \simeq$ $\operatorname{Det}_{\tau}^{+}\left(G_{T}\right)^{(0)}$.

\section{§2.2. Projective invariance of a transverse density}

In this paper we consider foliations satisfying the following two conditions. The first one is:

(PI) There exists a nonvanishing smooth density $\omega \in \Gamma\left(T\right.$, $\left.\operatorname{Det}_{\tau}^{+}(T)\right)$ which is projectively invariant under $G_{T}$.

When the groupoid $G_{T}$ satisfies the condition (PI), the subgroupoid $G_{T}^{(u)}=$ $\left\{\gamma \in G_{T} \mid \delta^{(\omega)}(\gamma)=1\right\}$ is an open subset of $G_{T}$. The second condition concerns the ergodicity of this subgroupoid. 
(UE) The action of $G_{T}^{(u)}$ is ergodic with respect to the Lebesgue measure class on $T$.

Remark 2. If $G_{T}$ and $\omega$ satisfy the condition (PI), so do $G_{S}$ and $\left.\omega\right|_{S}$ for any open subset $S$ of $T$. Similarly, if they satisfy (UE), the groupoid $G_{S}$ is Morita equivalent to $G_{T}$ via the groupoid bimodule

$$
G_{T}^{S}=\{\gamma \in G \mid r(\gamma) \in S, s(\gamma) \in T\}
$$

endowed with a left action of $G_{S}$ and a right action of $G_{T}$. An analogous statement holds for $G_{S}^{(u)}$ and $G_{T}^{(u)}$.

Remark 3. By imposing the condition (UE), we exclude the following kind of 'false' examples. When $M=\mathbb{R}$ and $F$ is given by TM itself, the countable subset $T=\mathbb{Z}$ of $M$ is a transversal for $F$. Then a transverse density over $T$ is equivalent to a sequence of numbers indexed by integers. Hence any choice of density on $T$ satisfies the assumption (PI). The associated foliation algebra is the convolution algebra in one real variable, which should be regarded as a trivial object in the category of operator algebras.

Remark 4. As a consequence of (UE), $C_{r}^{*}\left(G_{T}\right)$ and $C_{r}^{*}\left(G_{T}^{(u)}\right)$ are simple and the relative commutant of $C_{r}^{*}\left(G_{T}^{(u)}\right)$ in $W(M ; F)$ is trivial.

Given a transversal $T$, let $X_{T}$ denote the closure of the image of the module morphism $\delta^{(\omega)}: G_{T} \rightarrow \mathbb{R}_{>0}$. We have

$$
X_{T}=\operatorname{Sp}\left(\sigma^{(\omega)}\right)
$$

by $(2)$. Let $S(W(M ; F))$ denote the $S$-invariant of $W(M ; F)[\mathrm{C} 1]$.

Lemma 5. Suppose that $G_{T}$ and $\omega$ satisfy the conditions (PI) and (UE). Then $S(W(M ; F)) \cap \mathbb{R}_{>0}$ is equal to $X_{T}$.

Proof. The centralizer algebra $W(M ; F)^{\phi_{\omega}}$ is identified with the groupoid von Neumann algebra $W\left(G_{T}^{(u)}\right)$, which is a factor by $(\mathrm{UE})$. Then $\Gamma\left(\sigma^{(\omega)}\right)=\operatorname{Sp}\left(\sigma^{(\omega)}\right)$ $=X_{T}$ by $[\mathrm{C} 1$, Proposition 2.2.2(c)], which proves the assertion.

Example 1. A foliation $F$ is said to be transversely affine when there exists a covering of $M$ by some foliation charts with respect to which the holonomy maps become affine transformations. Suppose that $T$ is a transversal contained in a foliation chart

$$
U \simeq\left\{\left(x_{1}, \ldots, x_{q}, y_{1}, \ldots, y_{p}\right) \in \mathbb{R}^{p} \times \mathbb{R}^{q}\right\}
$$


of $F$ such that the holonomy transformations are affine with respect to the transverse coordinate system $\left(x_{1}, \ldots, x_{q}\right)$. Then the transverse volume form

$$
|d x|=\left|d x_{1} \wedge \cdots \wedge d x_{q}\right|
$$

gives a projectively invariant density on $T$.

Example 2. Let $A$ be a hyperbolic matrix in $\mathrm{SL}_{2}(\mathbb{Z})$. It defines a homeomorphism of $\mathbb{T}^{2}=\mathbb{R}^{2} / \mathbb{Z}^{2}$. The associated mapping cone $M=M_{A} \mathbb{T}^{2}$ admits an Anosov foliation $F$. Specifically, let $u$ and $v$ be eigenvectors of $A$, associated with eigenvalues $\lambda<1<\lambda^{-1}$. Then the subspace of TM spanned by the image of $u$ and the suspension flow is integrable. We obtain a hyperfinite factor of type $\mathrm{III}_{\lambda}$ as the associated von Neumann algebra [B2].

Example 3. Let $X$ be the cosphere bundle $S^{*} \Sigma_{g}$ of an oriented closed Riemannian surface of genus $g>1$. Then $X$ admits a foliation $F$ of dimension 2 generated by the holocyclic flow and the geodesic flow. If one takes an embedding of $\pi_{1}\left(\Sigma_{g}\right)$ into $\mathrm{SL}_{2}(\mathbb{R})$ and identify $X$ with $\mathrm{SL}_{2}(\mathbb{R}) / \pi_{1}\left(\Sigma_{g}\right)$, then $F$ is generated by the left actions of the following matrices:

$$
A_{t}=\left[\begin{array}{ll}
1 & t \\
0 & 1
\end{array}\right], \quad B_{t}=\left[\begin{array}{cc}
e^{t} & 0 \\
0 & e^{-t}
\end{array}\right]
$$

When $T$ is transversal to $F$, left multiplication by

$$
C_{t}=\left[\begin{array}{ll}
1 & 0 \\
t & 1
\end{array}\right]
$$

gives a projectively invariant coordinate on $T$.

\section{§2.3. Transverse metric trivialization}

By assuming the condition (PI), we are limiting our considerations to a particular class of foliations with some tame transverse measure structure, but such a foliation does not need to possess a projectively invariant transverse metric structure. The lack of such a metric is an obstacle to extending cyclic cocycles on the smooth convolution algebra to the $\mathrm{C}^{*}$-algebra of $G_{T}$. To remedy this, we introduce the groupoid $\operatorname{Met}_{\tau}\left(G_{T}\right)$ of transverse metric trivializations and work with cyclic cocycles defined on its groupoid algebra as in [C7].

For each $x \in M$, let $\operatorname{Met}_{\tau x}$ denote the set of all strictly positive quadratic forms on the vector space $\tau_{x}$. By functoriality there is a natural action of $G_{T}$ on the manifold $\operatorname{Met}_{\tau}(T)=\bigcup_{x \in T} \operatorname{Met}_{\tau x}$. Let $\operatorname{Met}_{\tau}\left(G_{T}\right)$ denote the associated groupoid. 
The module map (1) induces a groupoid homomorphism $\delta^{(\omega)}$ from $\operatorname{Met}_{\tau}\left(G_{T}\right)$ to $\mathbb{R}_{>0}$ by the composition of the natural projection $\operatorname{Met}_{\tau}\left(G_{T}\right) \rightarrow G_{T}$ and $\delta^{(\omega)}$ on $G_{T}$.

On one hand, any density $\omega$ defines a subset of the total space of $\operatorname{Det}_{\tau}^{+}(M)$ as its image. On the other hand, there is a natural surjection $\operatorname{Met}_{\tau}(M) \rightarrow \operatorname{Det}_{\tau}^{+}(M)$. We let $\operatorname{SMet}_{\tau}^{(\omega)}(M)$ denote the inverse image of $\omega$ in $\operatorname{Met}_{\tau}(M)$. When $\gamma \in G_{T}$ and $\xi \in \operatorname{SMet}_{\tau}^{(\omega)}(T)_{s \gamma}$, the element

$$
\operatorname{SMet}_{\tau}^{(\omega)}(\gamma) \xi=\delta^{(\omega)}(\gamma)^{-1} \operatorname{Met}_{\tau}(\gamma) \xi
$$

is in $\operatorname{SMet}_{\tau}^{(\omega)}(T)_{r \gamma}$. It follows that $\gamma \mapsto \operatorname{SMet}_{\tau}^{(\omega)}(\gamma)$ defines an action of $G_{T}$ on $\operatorname{SMet}_{\tau}^{(\omega)}(T)$. Let us denote $\operatorname{SMet}_{\tau}^{(\omega)}\left(G_{T}\right)=G_{T} \ltimes_{\operatorname{SMet}_{\tau}^{(\omega)}} \operatorname{SMet}_{\tau}^{(\omega)}(T)$. Since the fiber of the projection $\operatorname{SMet}_{\tau}^{(\omega)}(T) \rightarrow T$ admits a canonical spin structure, one has the natural isomorphism between the $K$-groups of $C_{r}^{*}\left(\operatorname{SMet}_{\tau}^{(\omega)}\left(G_{T}\right)\right)$ and $C_{r}^{*}\left(G_{T}\right)$.

The modular function $\delta^{(\omega)}$ on $G_{T}$ lifts to a function (again denoted by $\delta^{(\omega)}$ by abuse of notation) on $\operatorname{SMet}_{\tau}^{(\omega)}\left(G_{T}\right)$. It defines a 1-parameter group $\sigma^{(\omega)}$ on $C_{c}^{\infty}\left(\operatorname{SMet}_{\tau}^{(\omega)} G_{T}\right)$. We have an embedding

$$
C_{c}^{\infty}\left(\operatorname{Met}_{\tau} G_{T}\right) \rightarrow \mathbb{R} \ltimes_{\sigma^{(\omega)}} C_{c}^{\infty}\left(\operatorname{SMet}_{\tau}^{(\omega)} G_{T}\right)
$$

with dense image in the smooth crossed product analogous to (3).

\section{$\S 3$. Periodic action and cyclic cocycles}

Suppose that $\sigma$ is an action of $\mathbb{T}$ on a $\mathrm{C}^{*}$-algebra $B$. For each $n \in \mathbb{Z}$, let $B_{n}$ denote the spectral subspace $\left\{x \in B \mid \sigma_{t}(x)=e^{2 \pi i n t} x\right\}$. We let $A$ denote the fixed point algebra $B_{0}$ of $\sigma$.

The dual action $\hat{\sigma}$ of $\mathbb{Z}$ on $\mathbb{T} \ltimes_{\sigma} B$ induces the Takesaki-Takai duality

$$
\mathbb{Z} \ltimes_{\hat{\sigma}} \mathbb{T} \ltimes_{\sigma} B \simeq K K B
$$

The Pimsner-Voiculescu exact sequence [PV] for the automorphism $\hat{\sigma}$ of $\mathbb{T} \ltimes_{\sigma} B$ is given by the 6 -term exact sequence

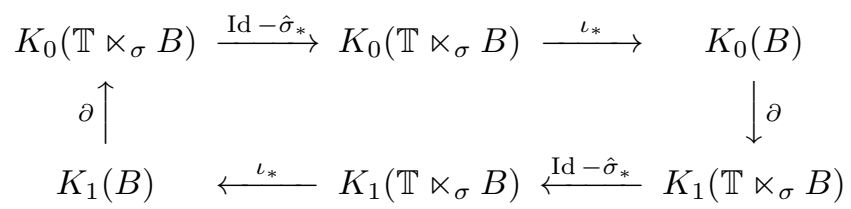

where $\iota^{*}$ is induced by the inclusion homomorphism $\iota: \mathbb{T} \ltimes_{\sigma} B \rightarrow \mathbb{Z} \ltimes_{\hat{\sigma}} \mathbb{T} \ltimes_{\sigma} B$ and the natural isomorphism $K_{*}\left(\mathbb{Z} \ltimes_{\hat{\sigma}} \mathbb{T} \ltimes_{\sigma} B\right) \simeq K_{*}(B)$.

The action $\sigma$ may be regarded as an action of $\mathbb{R}$ via the surjection $\mathbb{R} \rightarrow \mathbb{R} / \mathbb{Z}$ $\simeq \mathbb{T}$. Let $\Psi$ be the $*$-algebra homomorphism from $\mathbb{R} \ltimes_{\sigma} B$ to $\mathbb{T} \ltimes_{\sigma} B$ characterized 
as the unique extension of the mapping

$$
\Psi(f)_{t}=\sum_{m \in \mathbb{Z}} f(t+m)
$$

from $L^{1}(\mathbb{R} ; B) \subset \mathbb{R} \ltimes_{\sigma} B$ to $L^{1}(\mathbb{T} ; B) \subset \mathbb{T} \ltimes_{\sigma} B$.

Lemma 6. The map $\Psi_{*}: K_{*}\left(\mathbb{R} \ltimes_{\sigma} B\right) \rightarrow K_{*}\left(\mathbb{T} \ltimes_{\sigma} B\right)$ induced by $\Psi$ is identified with the map $\partial$ in (9) via the Connes-Thom isomorphism $K_{*}(B) \simeq K_{*+1}\left(\mathbb{R} \ltimes_{\sigma} B\right)$ and the natural isomorphism $K_{*}\left(\mathbb{T} \ltimes_{\sigma} B\right) \simeq K_{*}(A)$ induced by (8).

Proof. The crossed product $\mathbb{R} \ltimes_{\sigma} B$ is identified with the mapping torus

$$
M_{\hat{\sigma}}\left(\mathbb{T} \ltimes_{\sigma} B\right)=\left\{f \in C\left(\mathbb{R} ; \mathbb{T} \ltimes_{\sigma} B\right) \mid f_{t+1}=\hat{\sigma}\left(f_{t}\right)\right\} .
$$

Under this identification the homomorphism $\Psi$ corresponds to the evaluation map $f \mapsto f_{0}$ from $M_{\hat{\sigma}}\left(\mathbb{T} \ltimes_{\sigma} B\right)$ to $\mathbb{T} \ltimes_{\sigma} B$. Then Connes's proof [C3, pp. 48-49] of the Pimsner-Voiculescu exact sequence implies the assertion.

Let $\mathcal{B}$ be a locally convex algebra over $\mathbb{C}$. Let $\Omega(\mathcal{B})$ be the universal differential graded algebra over $\mathcal{B}$. It is the direct sum $\bigoplus_{n \in \mathbb{N}} \Omega_{n}(\mathcal{B})$, where

$$
\Omega_{0}(\mathcal{B})=\mathcal{B}, \quad \Omega_{n}(\mathcal{B})=\mathcal{B}^{\otimes n} \oplus \mathcal{B}^{\otimes n+1} \quad(n>0) .
$$

An element $\left(a^{1}, \ldots, a^{n}\right) \oplus\left(b^{0}, \ldots, b^{n}\right) \in \Omega_{n}(\mathcal{B})$ is understood to represent the $n$ form $d a^{1} \cdots d a^{n}+b^{0} d b^{1} \cdots d b^{n}$. Thus the differential $d: \Omega_{n}(\mathcal{B}) \rightarrow \Omega_{n+1}(\mathcal{B})$ is given by the identity map on the factor $\mathcal{B}^{\otimes n+1}$ on both sides. The algebra structure on $\Omega(\mathcal{B})$ can be determined using the Leibniz rule for $d$.

Let $\kappa$ denote the operator

$$
f^{0} \otimes \cdots \otimes f^{n} \mapsto(-1)^{n} f^{n} \otimes f^{0} \otimes f^{1} \otimes \cdots \otimes f^{n-1}
$$

on $\mathcal{B}^{\otimes n+1}$. It satisfies $\kappa^{n+1}=1$. Next, there is the $b^{\prime}$-operator

$$
b^{\prime} \psi\left(f^{0}, \ldots, f^{n}\right)=\sum_{k=0}^{n-1}(-1)^{k} \psi\left(f^{0}, \ldots, f^{k} f^{k+1}, \ldots, f^{n}\right)
$$

and the Hochschild coboundary operator $b$

$$
b \psi\left(f^{0}, \ldots, f^{n}\right)=b^{\prime} \psi\left(f^{0}, \ldots, f^{n}\right)+(-1)^{n} \psi\left(f^{n} f^{0}, f^{1}, \ldots, f^{n-1}\right),
$$

from $\left(\mathcal{B}^{\otimes n}\right)^{\prime}$ to $\left(\mathcal{B}^{\otimes n+1}\right)^{\prime}$. The cyclic cocycles are precisely the ones in the joint kernel of $1-\kappa$ and $b$. One also has the $S$-operator of Connes [C6, Section I.4].

As in the $\mathrm{C}^{*}$-algebraic setting before, let $\sigma$ be an action of $\mathbb{T}$ on $\mathcal{B}$. In the rest of this section we let $\phi$ denote a $\sigma$-invariant cyclic $n$-cocycle on $\mathcal{B}$. One obtains the following two new cyclic cocycles. 
We let $\mathbb{R} \ltimes_{\sigma} \mathcal{B}$ denote the space of Schwartz class functions from $\mathbb{R}$ to $\mathcal{B}$ endowed with the convolution product. The first cocycle, $\hat{\phi}$, is the cyclic $n$-cocycle on $\mathbb{R} \ltimes_{\sigma} \mathcal{B}$ given by

$$
\left\langle\hat{\phi}, f^{0} d f^{1} \cdots d f^{n}\right\rangle=\int_{\sum_{j=0}^{n} t_{j}=0} \phi\left(f_{t_{0}}^{0}, \sigma_{t_{0}}\left(f_{t_{1}}^{1}\right), \ldots, \sigma_{\sum_{j=0}^{n-1} t_{j}}\left(f_{t_{n}}^{n}\right) .\right.
$$

Let $D$ be the generator of the action $\sigma$. The second cocycle, $i_{D} \phi$, is the cyclic $n+1$-cocycle on $\mathcal{B}$ defined by

$$
i_{D} \phi\left(f^{0}, \ldots, f^{q^{\prime}+1}\right)=\sum_{j=1}^{q^{\prime}}(-1)^{j-1}\left\langle\phi, f^{0} d f^{1} \cdots D\left(f^{j}\right) \cdots d f^{q^{\prime}+1}\right\rangle .
$$

There is also a boundedness condition for $\phi$, namely the $n$-trace property, which depends on the choice of a norm $\|\cdot \mid\|$ on $\mathcal{B}$. Specifically, $\phi$ is said to be an $n$-trace if for any elements $\left(g^{i}\right)_{i=1}^{n}$ of $B$, there exists a constant $C$ satisfying

$$
\left|\tau_{\phi}\left(f^{0} g^{1} d f^{1} \cdots g^{n} d f^{n}\right)\right| \leq C \prod_{i=1}^{n}\|\| g^{i}\|\| \quad\left(f^{i} \in \mathcal{B}\right)
$$

where $\tau_{\phi}$ is the associated closed graded trace on $\Omega^{*}(\mathcal{B})$. If $\phi$ satisfies the $n$-trace condition, the map $K_{n}(\mathcal{B}) \rightarrow \mathbb{C}$ induced by $\phi$ can be extended to $K_{n}(\mathrm{~B})$, where $\mathrm{B}$ is the completion of $\mathcal{B}$ with respect to $\|\cdot \cdot\| \cdot$. Moreover, when this is the case, $\hat{\phi}$ and $i_{D} \phi$ are also an $n$-trace and an $(n+1)$-trace on $\mathbb{R} \ltimes \mathcal{B}$ and $\mathcal{B}$ respectively [C8, Section 3.6. $\beta$ ]. When the norm $\|\cdot \mid\|$ is chosen appropriately, the algebra $\mathrm{B}$ becomes a spectral subalgebra of the initial $\mathrm{C}^{*}$-algebra $B$ and has the same $K$-groups.

The action $\sigma$ on $\mathcal{B}$ has a canonical extension to the algebra $\Omega(\mathcal{B})$, and the closed trace $\tau_{\phi}$ on $\Omega(\mathcal{B})$ associated with $\phi$ becomes invariant under this extended action. Given an element $f$ of $\mathbb{R} \ltimes_{\sigma} \mathcal{B}$, we let $d f$ denote the 'pointwise derivation' $t \mapsto d\left(f_{t}\right)$, regarded as an element in $\mathbb{R} \ltimes_{\sigma} \Omega(\mathcal{B})$.

For ease of notation, we introduce several notations which are only used in this section. Given the standard coordinate $\left(t_{0}, \ldots, t_{n}\right)$ on $\mathbb{R}^{n+1}$ and an integer $1 \leq j<n$, let $s_{j}$ denote the quantity $\sum_{0 \leq k<j} t_{k}$. In addition, given elements $f^{0}, \ldots, f^{n}$ of $\mathbb{R} \ltimes_{\sigma} \mathcal{B}$, let $g_{j}$ denote the function $\sigma_{s_{j}}\left(f_{t_{j}}^{j}\right)$ from $\mathbb{R}^{j}$ to $\mathcal{B}$ for $0 \leq j \leq n$.

Lemma 7. For each $m \in \mathbb{Z}$, the functional

$$
\hat{\phi}_{m}\left(f^{0}, \ldots, f^{n}\right)=\tau_{\phi}\left(\left(f^{0} d f^{1} \cdots d f^{n}\right)_{m}\right)=\int_{\left(t_{j}\right) \in \mathbb{R}^{n}, \sum_{j=0}^{n} t_{j}=m} \phi\left(g_{0}, \ldots, g_{n}\right)
$$

is a Hochschild $n$-cocycle over $\mathbb{R} \ltimes_{\sigma} \mathcal{B}$.

Proof. The $\sigma$-invariance of $\phi$ and the assumption that $\sigma$ is periodic imply

$$
\phi\left(f_{t_{n+1}}^{n+1} \sigma_{t_{n+1}}\left(g_{0}\right), \ldots, \sigma_{t_{n+1}}\left(g_{n}\right)\right)=\phi\left(g_{n+1} g_{0}, g_{1}, \ldots, g_{n}\right)
$$


whenever $\sum_{j=0}^{n+1} t_{j}=m$. Thus, $b \hat{\phi}_{m}\left(f^{0}, \ldots, f^{n+1}\right)$ is equal to

$$
\int_{\sum_{j=0}^{n+1} t_{j}=m} \sum_{k=0}^{n}(-1)^{k} \phi\left(g_{0}, g_{1}, \ldots, g_{j} g_{j+1}, \ldots, g_{n}\right)+(-1)^{n+1} \phi\left(g_{n+1} g_{0}, g_{1}, \ldots, g_{n}\right) \text {. }
$$

By the Hochschild cocycle condition on $\phi$, the integrand above vanishes. Hence $b \hat{\phi}_{m}=0$.

Remark 8. The Hochschild cocycle $\hat{\phi}_{m}$ satisfies the cyclicity condition when $m=0$, but otherwise the cyclicity is not guaranteed.

Let $\hat{D}$ be the derivation $\hat{D}(f)_{t}=t f_{t}$ on $\mathbb{R} \ltimes \mathcal{B}$. It is the generator of the dual action $\hat{\sigma}$ of $\mathbb{R}$ on $\mathbb{R} \ltimes_{\sigma} \mathcal{B}$.

Lemma 9. For each $m \in \mathbb{Z}$, the multilinear functional

$$
\eta_{m}\left(f^{0}, \ldots, f^{n+1}\right)=\sum_{k=1}^{n+1}(-1)^{k+1} \tau_{\phi}\left(\left(f^{0} d f^{1} \cdots \hat{D}\left(f^{k}\right) \cdots d f^{n+1}\right)_{m}\right)
$$

is an $(n+1)$-Hochschild cocycle over $\mathbb{R} \ltimes_{\sigma} \mathcal{B}$.

Proof. By definition, $\eta_{m}$ is the action $\hat{D} \# \hat{\phi}_{m}$ of the derivation $\hat{D}$ on the Hochschild cocycle $\hat{\phi}_{m}$ [C8, Remark 3.2.30.b]. Hence it satisfies the Hochschild coboundary relation.

Lemma 10. For any integers $n$ and $m$, one has $\left(1-\kappa^{*}\right) \eta_{m}=(-1)^{n} m b^{\prime} \hat{\phi}_{m}$.

Proof. For each $1 \leq k \leq n$, the term $\tau_{\phi}\left(\left(f^{0} d f^{1} \cdots \hat{D}\left(f^{k}\right) \cdots d f^{n+1}\right)_{m}\right)$ contributes

$$
\int_{t_{0}+\cdots+t_{n}=m} t_{k} \sum_{j=0}^{k-1}(-1)^{k-j-1} \phi\left(g_{0}, \ldots, g_{j} g_{j+1}, \ldots, g_{n}\right) .
$$

Hence $\eta_{m}\left(f^{0}, \ldots, f^{n}\right)$ is the sum of these terms. Similarly, $\kappa^{*} \eta_{m}\left(f^{0}, \ldots, f^{n}\right)$ is the sum of

$$
\begin{aligned}
& (-1)^{n} \int_{t_{0}+\cdots+t_{n}=m}\left[t_{k} \phi\left(f_{t_{n}}^{n} \sigma_{t_{n}}\left(g_{0}\right), \sigma_{t_{n}}\left(g_{1}\right), \ldots, \sigma_{t_{n}}\left(g_{n-1}\right)\right)\right. \\
& \left.+t_{k} \sum_{j=0}^{k-1}(-1)^{k-j-1} \phi\left(f_{t_{n}}^{n}, \sigma_{t_{n}}\left(g_{0}\right), \ldots, \sigma_{t_{n}}\left(g_{j}\right) \sigma_{t_{n}}\left(g_{j+1}\right), \ldots, \sigma_{t_{n}}\left(g_{n-1}\right)\right)\right]
\end{aligned}
$$

for $0 \leq k \leq n-1$.

First, by the cyclicity and $\sigma$-invariance of $\phi$, one has

$$
\begin{aligned}
(-1)^{n}(-1)^{k-j-1} \phi\left(f_{t_{n}}^{n}, \sigma_{t_{n}}\left(g_{0}\right), \ldots,\right. & \left.\sigma_{t_{n}}\left(g_{j}\right) \sigma_{t_{n}}\left(g_{j+1}\right), \ldots, \sigma_{t_{n}}\left(g_{n-1}\right)\right) \\
& =(-1)^{k-j-1} \phi\left(g_{0}, \ldots, g_{j} g_{j+1}, \ldots, g_{n}\right)
\end{aligned}
$$


for each $j$ when $t_{0}+\cdots+t_{n}=m$. This shows that

$$
\begin{aligned}
& \sum_{j=0}^{k-1}(-1)^{k-j-1} \phi\left(g_{0}, \ldots, g_{j} g_{j+1}, \ldots, g_{n}\right) \\
& -(-1)^{n} \sum_{j=0}^{k-1}(-1)^{k-j-1} \phi\left(f_{t_{n}}^{n}, \sigma_{t_{n}}\left(g_{0}\right), \ldots, \sigma_{t_{n}}\left(g_{j}\right) \sigma_{t_{n}}\left(g_{j+1}\right), \ldots, \sigma_{t_{n}}\left(g_{n-1}\right)\right)
\end{aligned}
$$

vanishes for $1 \leq k \leq n-1$.

Next, by the Hochschild cocycle condition on $\phi$, one has

$$
\begin{aligned}
& t_{n} \sum_{j=0}^{n-1}(-1)^{n-j-1} \phi\left(g_{0}, \ldots, g_{j} g_{j+1}, \ldots, g_{n}\right) \\
& \quad=(-1)^{n+1} t_{n} \phi\left(f_{t_{n}}^{n}, \sigma_{t_{n}}\left(g_{0}\right), \ldots, \sigma_{t_{n}}\left(g_{j}\right) \sigma_{t_{n}}\left(g_{j+1}\right), \ldots, \sigma_{t_{n}}\left(g_{n-1}\right)\right) .
\end{aligned}
$$

Combining these, $\left(1-\kappa^{*}\right) \eta_{m}\left(f^{0}, \ldots, f^{n}\right)$ reduces to

$$
\int_{\sum_{j=0}^{3} t_{j}=m}\left(\sum_{j=0}^{n} t_{j}\right) \phi\left(f_{t_{n}}^{n} \sigma_{t_{n}}\left(f_{t_{0}}^{0}\right), \sigma_{t_{n}+s_{1}}\left(f_{t_{1}}^{1}\right), \ldots, \sigma_{t_{n}+s_{n-1}}\left(f_{t_{n-1}}^{n-1}\right)\right),
$$

which proves the assertion in this case.

Let us illustrate the content of Lemma 10 for $n=1$. By definition of $\eta_{m}$, one has

$$
\begin{aligned}
\eta_{m}\left(f^{0}, f^{1}, f^{2}\right) & =\left\langle\tau_{\phi},\left(f^{0} \hat{D}\left(f^{1}\right) d f^{2}-f^{0} d f^{1} \hat{D}\left(f^{2}\right)\right)_{m}\right\rangle \\
& =\left\langle\tau_{\phi},\left(f^{0} \hat{D}\left(f^{1}\right) d f^{2}-f^{0} d\left(f^{1} \hat{D}\left(f^{2}\right)\right)+f^{0} f^{1} d \hat{D}\left(f^{2}\right)\right)_{m}\right\rangle,
\end{aligned}
$$

which is equal to

$$
\begin{aligned}
\int_{t_{0}+t_{1}+t_{2}=m}\left[t_{1} \phi\left(f_{t_{0}}^{0} \sigma_{t_{0}}\left(f_{t_{1}}^{1}\right), \sigma_{t_{0}+t_{1}}\left(f_{t_{2}}^{2}\right)\right)\right. \\
\left.-t_{2} \phi\left(f_{t_{0}}^{0}, \sigma_{t_{0}}\left(f_{t_{1}}^{1}\right) \sigma_{t_{0}+t_{1}}\left(f_{t_{2}}^{2}\right)\right)+t_{2} \phi\left(f_{t_{0}}^{0} \sigma_{t_{0}}\left(f_{t_{1}}^{1}\right), \sigma_{t_{0}+t_{1}}\left(f_{t_{2}}^{2}\right)\right)\right] .
\end{aligned}
$$

Then

$$
\left(1-\kappa^{*}\right) \eta_{m}\left(f^{0}, f^{1}, f^{2}\right)=\eta_{m}\left(f^{0}, f^{1}, f^{2}\right)-\eta_{m}\left(f^{2}, f^{0}, f^{1}\right)
$$

can be computed as

$$
\begin{aligned}
\int_{t_{0}+t_{1}+t_{2}=m}\left[t_{1} \phi\left(f_{t_{0}}^{0} \sigma_{t_{0}}\left(f_{t_{1}}^{1}\right), \sigma_{t_{0}+t_{1}}\left(f_{t_{2}}^{2}\right)\right)-t_{2} \phi\left(f_{t_{0}}^{0}, \sigma_{t_{0}}\left(f_{t_{1}}^{1}\right) \sigma_{t_{0}+t_{1}}\left(f_{t_{2}}^{2}\right)\right)\right. \\
+t_{2} \phi\left(f_{t_{0}}^{0} \sigma_{t_{0}}\left(f_{t_{1}}^{1}\right), \sigma_{t_{0}+t_{1}}\left(f_{t_{2}}^{2}\right)\right)-t_{0} \phi\left(f_{t_{2}}^{2} \sigma_{t_{2}}\left(f_{t_{0}}^{0}\right), \sigma_{t_{2}+t_{0}}\left(f_{t_{1}}^{1}\right)\right) \\
\left.+t_{1} \phi\left(f_{t_{2}}^{2}, \sigma_{t_{2}}\left(f_{t_{0}}^{0}\right) \sigma_{t_{2}+t_{0}}\left(f_{t_{1}}^{1}\right)\right)-t_{1} \phi\left(f_{t_{2}}^{2} \sigma_{t_{2}}\left(f_{t_{0}}^{0}\right), \sigma_{t_{2}+t_{0}}\left(f_{t_{1}}^{1}\right)\right)\right] .
\end{aligned}
$$


By the cyclicity condition $\phi=\kappa^{*} \phi$ and the $\sigma$-invariance of $\phi$, one has

$$
t_{1} \phi\left(f_{t_{0}}^{0} \sigma_{t_{0}}\left(f_{t_{1}}^{1}\right), \sigma_{t_{0}+t_{1}}\left(f_{t_{2}}^{2}\right)\right)=-t_{1} \phi\left(f_{t_{2}}^{2}, \sigma_{t_{2}}\left(f_{t_{0}}^{0}\right) \sigma_{t_{2}+t_{0}}\left(f_{t_{1}}^{1}\right)\right) .
$$

On the other hand, the Hochschild cocycle condition $b \phi=0$ implies

$$
\begin{aligned}
t_{2}\left\{\phi\left(f_{t_{0}}^{0}, \sigma_{t_{0}}\left(f_{t_{1}}^{1}\right) \sigma_{t_{0}+t_{1}}\left(f_{t_{2}}^{2}\right)\right)-\phi\left(f_{t_{0}}^{0} \sigma_{t_{0}}\left(f_{t_{1}}^{1}\right), \sigma_{t_{0}+t_{1}}\left(f_{t_{2}}^{2}\right)\right)\right\} & =t_{2} \phi\left(f_{t_{2}}^{2} \sigma_{t_{2}}\left(f_{t_{0}}^{0}\right), \sigma_{t_{2}+t_{0}}\left(f_{t_{1}}^{1}\right)\right) .
\end{aligned}
$$

Combining these, one obtains

$$
\left(1-\kappa^{*}\right) \eta_{m}\left(f^{0}, f^{1}, f^{2}\right)=\int_{t_{0}+t_{1}+t_{2}=m}(-m) \phi\left(f_{t_{2}}^{2} \sigma_{t_{2}}\left(f_{t_{0}}^{0}\right), \sigma_{t_{2}+t_{0}}\left(f_{t_{1}}^{1}\right)\right),
$$

which implies $\left(1-\kappa^{*}\right) \eta_{m}=-m b^{\prime} \hat{\phi}_{m}$ when $n=1$.

Analogously to the $\mathbb{R}$-crossed product case, we have the dual cyclic cocycle $\hat{\phi}^{\mathbb{T}}$ on $\mathbb{T} \ltimes_{\sigma} \mathcal{B}$ defined by

$$
\hat{\phi}^{\mathbb{T}}\left(f^{0}, \ldots, f^{n}\right)=\tau_{\phi}\left(\left(f^{0} d f^{1} \cdots d f^{n}\right)_{m}\right)=\int_{\left(t_{j}\right) \in \mathbb{T}^{n}, \sum_{j=0}^{n} t_{j}=m} \phi\left(g_{0}, \ldots, g_{n}\right) .
$$

When $\mathcal{B}$ is Fréchet, the formula (6) defines a homomorphism from $\mathbb{R} \ltimes_{\sigma} \mathcal{B}$ to $\mathbb{T} \ltimes_{\sigma} \mathcal{B}$, which we denote again by $\Psi$. Then the pullback cocycle on $\mathbb{R} \ltimes_{\sigma} \mathcal{B}$ is represented as

$$
\Psi^{*}\left(\hat{\phi}^{\mathbb{T}}\right)\left(f^{0}, \ldots, f^{n}\right)=\sum_{m \in \mathbb{Z}}\left\langle\tau_{\phi},\left(f^{0} d f^{1} \cdots d f^{n}\right)_{m}\right\rangle=\sum_{m \in \mathbb{Z}} \hat{\phi}_{m}\left(f^{0}, \ldots, f^{n}\right) .
$$

We note that this pullback does not agree with $\hat{\phi}_{0}$. However, they define the same periodic cyclic cohomology class, as is seen in the next theorem.

Theorem 1. Let $\sigma$ be an action of $\mathbb{T}$ on a Fréchet algebra $\mathcal{B}$. When $\phi$ is a $\sigma$-invariant cyclic $n$-cocycle on $\mathcal{B}$, the cyclic cocycles $\hat{\phi}$ and $\Psi^{*}\left(\hat{\phi}^{\mathbb{T}}\right)$ are cohomologous in $\mathrm{HC}^{n+2}\left(\mathbb{R} \ltimes_{\sigma} \mathcal{B}\right)$.

Proof. We know that $m S \hat{\phi}_{m}$ is a cyclic coboundary for any $m$ by Lemmas 9 and 10, and the $(b, B)$-bicomplex argument as in [C6, Lemma 29; C9]. By (7), $\Psi^{*}\left(\hat{\phi}^{\mathbb{T}}\right)$ becomes cohomologous to $\hat{\phi}_{0}$ after applying $S$. Since $\hat{\phi}_{0}=\hat{\phi}$, this proves the assertion.

We now combine the considerations of the $\mathrm{C}^{*}$-algebraic setting and the Fréchet algebra setting to obtain the main result of this section. Let $\sigma$ be an action of $\mathbb{T}$ on a $\mathrm{C}^{*}$-algebra $B$. Moreover, we assume that it acts smoothly on a spectral subalgebra $\mathcal{B}$ of $B$ with a Fréchet topology. 
Corollary 11. Let $\sigma, B$, and $\mathcal{B}$ be as above, and let $\phi$ be a $\sigma$-invariant $n$-trace on $\mathcal{B}$. Then for any $x \in K_{*}(\mathcal{B})$ one has $\left\langle i_{D} \phi, x\right\rangle=\left\langle\hat{\phi}^{\mathbb{T}}, \partial(x)\right\rangle$ with $\partial$ the homomorphism in (5).

Proof. The cocycles $\hat{\phi}$ and $i_{D} \phi$ induce the same map (implicitly proved in [ENN, Lemma 6.3]; see [Y, Proposition 14] for an explicit proof) via the identification given by the Connes-Thom isomorphism $\Phi: K_{*}\left(\mathbb{R} \ltimes_{\sigma} B\right) \rightarrow K_{*+1}(B)$. The assertion follows from Lemma 6 and Theorem 1.

When $\sigma$ has full strong spectrum, that is, $B_{-n} B_{n}=A$ for any $n$, we have the strong Morita equivalence

$$
\mathbb{T} \ltimes_{\sigma} B \simeq K K A
$$

This condition is satisfied when $A$ is simple and $B_{n} \neq\{0\}$ for every $n$, which is relevant to our setting in view of the condition (UE). When this is the case, the 6 -term exact sequence (5) for the action $\hat{\sigma}$ of $\mathbb{Z}$ on $\mathbb{T} \ltimes_{\sigma} B$ becomes

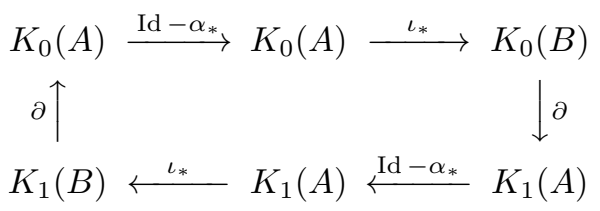

Corollary 12. Let $\sigma$ be an action of $\mathbb{R}$ on a Fréchet pre- $C^{*}$-algebra $\mathcal{B}$. Suppose that the spectrum of $\sigma$ is equal to $T \mathbb{Z} \subset \mathbb{R}$ for some real number $T$. Then, for any $x \in K_{*}(\mathcal{B})$ one has $\left\langle i_{D} \phi, x\right\rangle=T\langle\phi, \partial(x)\rangle$ with $\partial$ the homomorphism in (9).

Proof. When $R$ is an arbitrary positive real number, we have $i_{R D} \phi=R i_{D} \phi$. Hence we may assume that $T=2 \pi$. Thus we assume that $\sigma$ comes from a full spectrum action of $\mathbb{T}=\mathbb{R} / 2 \pi \mathbb{Z}$ on $\mathcal{B}$.

The cyclic cocycle $\left.\phi\right|_{\mathcal{A}}$ corresponds to the multilinear functional

$$
\begin{aligned}
\psi_{0}\left(f^{0}, \ldots, f^{n}\right) & =\left\langle\tau_{\phi}, E\left(\left(f^{0} d f^{1} \cdots d f^{n}\right)_{0}\right)\right\rangle \\
& =\int_{T} d t^{\prime} \int_{t_{0}+\cdots+t_{n}=0} d t_{0} \cdots d t_{n} \sigma_{t^{\prime}}^{*} \phi\left(f_{t_{0}}^{0}, \sigma_{s_{1}}\left(f_{t_{1}}^{1}\right), \ldots, \sigma_{s_{n}}\left(f_{t_{n}}^{n}\right)\right) \\
& =\int_{t_{0}+\cdots+t_{n}=0} \phi\left(f_{t_{0}}^{0}, \sigma_{s_{1}}\left(f_{t_{1}}^{1}\right), \ldots, \sigma_{s_{n}}\left(f_{t_{n}}^{n}\right)\right) d t_{0} \cdots d t_{n}
\end{aligned}
$$

on $\mathcal{S}(\mathbb{T} ; \mathcal{B}) \subset \mathbb{T} \ltimes_{\sigma} \mathcal{B}$ via the strong Morita equivalence (8).

Example 4. Let $\mathcal{B}$ be the Fréchet algebra $C^{\infty}(\mathbb{T})$ and $\sigma$ be the action of $\mathbb{T}$ on $\mathcal{B}$ induced by translation. There is a unique $\sigma$-invariant trace $\tau$ on $\mathcal{B}$, namely integration of functions with respect to the normalized Haar measure on $\mathbb{T}$. The fixed 
point algebra $\mathcal{A}$ is equal to $\mathbb{C}$, and the cyclic 1-cocycle $i_{D} \tau$ on $\mathcal{B}$ is equal to the bilinear functional

$$
i_{D} \tau\left(f^{0}, f^{1}\right)=\frac{1}{2 \pi i} \int_{\mathbb{T}} f^{0} d f^{1},
$$

defined as the invariant integral of 1-forms over the 1-dimensional manifold $\mathbb{T}$.

The 6 -term exact sequence (9) is the one induced by the extension of algebras $C_{0}(0,1) \rightarrow C(\mathbb{T}) \rightarrow \mathbb{C}$. The map induced by $\left.\tau\right|_{\mathbb{C}}$ on $K_{0}(\mathbb{C})$ and the one induced by $i_{D} \tau$ on $K_{1}\left(C_{0}(0,1)\right)$ indeed agree, as each of them sends generators of the corresponding $K$-group to \pm 1 .

Remark 13. Let $\alpha$ be an action of $\mathbb{Z}$ on a $\mathrm{C}^{*}$-algebra $A$. Suppose that there is a Fréchet subalgebra $\mathcal{A} \subset A$ which is invariant under $\alpha$. R. Nest $[\mathrm{N}]$ constructed, by means of a spectral sequence, a homomorphism $\partial^{*}: \operatorname{HP}^{*}(\mathcal{A}) \rightarrow \operatorname{HP}^{*+1}\left(\mathbb{Z} \ltimes_{\sigma} \mathcal{A}\right)$ which is transpose to the homomorphism $\partial$ of (5).

If $\phi$ is an $\alpha$-invariant cyclic $n$-cocycle on $\mathcal{A}$, then one has the dual cocycle $\hat{\phi}^{\mathbb{Z}}$ on $\mathbb{Z} \ltimes_{\alpha} \mathcal{A}$ defined by

$$
\hat{\phi}^{\mathbb{Z}}\left(f^{0}, \ldots, f^{n}\right)=\sum_{m_{0}+\cdots+m_{n}=0} \phi\left(f_{m_{0}}^{0}, \alpha_{m_{0}}\left(f_{m_{1}}^{1}\right), \ldots, \alpha_{m_{0}+\cdots+m_{n-1}}\left(f_{m_{n}}^{n}\right)\right) .
$$

This cocycle is invariant under the dual action $\hat{\alpha}$. Hence we obtain another cyclic $(n+1)$-cocycle $i_{D} \hat{\phi}^{\mathbb{Z}}$ on $\mathbb{Z} \ltimes_{\sigma} \mathcal{A}$, where $D$ is the generator of $\hat{\alpha}$.

In this setting Corollary 11 says that the image of $\phi$ under $\partial^{*}$ pairs with the $K$-group in the same way as $i_{D} \hat{\phi}^{\mathbb{Z}}$ does. The strong Morita equivalence (8) becomes the one between $\mathbb{Z} \ltimes_{\alpha} \mathcal{A}$ and the crossed product by the suspension flow on the mapping torus of $\alpha$. From this point of view, the correspondence of Corollary 11 is a generalization of the correspondence in Example 4.

\section{$\S 4$. Invariance of the fundamental cocycle}

We return to the setting of Section 2, so let $F$ be a foliation on $M$, and $T$ a transverse submanifold of $M$. Let $q^{\prime}$ denote the total dimension $q(q+1) / 2+q-1$ of $\operatorname{SMet}_{\tau}^{(\omega)}(T)$. We consider the transverse fundamental cocycle [C7] of the groupoid $\operatorname{SMet}_{\tau}^{(\omega)}\left(G_{T}\right)$. It is defined as the following cyclic $q^{\prime}$-cocycle:

$$
\phi\left(f^{0}, \ldots f^{q^{\prime}}\right)=\int_{\gamma_{0} \cdots \gamma_{q^{\prime}} \in \operatorname{SMet}_{\tau}^{(\omega)} T} f_{\gamma_{0}}^{0} d f_{\gamma_{1}}^{1} \cdots d f_{\gamma_{q^{\prime}}}^{q^{\prime}} .
$$

In the following we analyze the pairing of $\hat{\phi}$ with $K_{q^{\prime}}\left(\mathbb{R} \ltimes_{\sigma} C^{*} \operatorname{SMet}_{\tau}^{(\omega)} G_{T}\right)$ and its relation to the structure of the von Neumann algebra $W(M ; F)$. Following the method of [C7], we will construct an invariant complex measure on the flow of 
weights when the cyclic cocycle $\hat{\phi}$ pairs nontrivially with the $K$-group of $\mathbb{R} \ltimes_{\sigma^{(\omega)}}$ $C_{r}^{*}\left(\operatorname{SMet}_{\tau}^{(\omega)}\left(G_{T}\right)\right) \simeq C_{r}^{*}\left(\operatorname{Met}_{\tau}\left(G_{T}\right)\right)$.

Proposition 14. Suppose that the foliation $(M ; F)$, the transversal $T$, and the transverse density $\omega$ satisfy the condition $(\mathrm{PI})$. Then the cocycle $\phi$ of (10) is invariant under the 1-parameter group $\sigma_{t}$.

Proof. The derivative of $\sigma_{t}^{*} \phi$ is given by

$$
\begin{aligned}
& \frac{d \sigma_{t}^{*} \phi}{d t}\left(f^{0}, \ldots, f^{q^{\prime}}\right) \\
& =\sum_{1 \leq j \leq q^{\prime}} \int_{\gamma_{0} \cdots \gamma_{q^{\prime}} \in \operatorname{SMet}_{\tau}^{(\omega)} T} i f^{0}\left(\gamma_{0}\right) f^{j}\left(\gamma_{j}\right) d \log \left(\delta\left(\gamma_{j}\right)\right) d f^{1}\left(\gamma_{1}\right) \cdots \widetilde{d f^{j}\left(\gamma_{j}\right)} \cdots d f^{q^{\prime}}\left(\gamma_{q^{\prime}}\right) \\
& +\sum_{0 \leq j \leq q^{\prime}} \int_{\gamma_{0} \cdots \gamma_{q^{\prime}} \in \operatorname{SMet}_{\tau}^{(\omega)} T} \log \left(\delta\left(\gamma_{j}\right)\right) f^{0}\left(\gamma_{0}\right) d f^{1}\left(\gamma_{1}\right) \cdots d f^{q^{\prime}},
\end{aligned}
$$

where the tilde indicates omission of the corresponding term.

By assumption $(\mathrm{PI}), \delta(\gamma)$ is constant along the transverse movement. This implies $d \log \left(\delta\left(\gamma_{j}\right)\right)=0$ for each term in the first part of the right hand side. Furthermore, $\gamma_{0} \cdots \gamma_{q^{\prime}} \in \operatorname{SMet}_{\tau}^{(\omega)} T$ implies $\sum_{0 \leq j \leq q^{\prime}} \log \left(\delta\left(\gamma_{j}\right)\right)=0$. Consequently, the second part is also trivial.

Remark 15. Suppose that the codimension $q$ is equal to 1 . The Godbillon-Vey class of $(M ; F)$ can be obtained from the derivation of the transverse fundamental class with respect to the action of the modular automorphism group [C7, Lemma 7.6]. In particular, the Godbillon-Vey number has to be 0 under our assumption, but the von Neumann algebra can be of type III as in Example 2.

Connes [C7] showed that the fundamental cocycle of the groupoid $\operatorname{Met}_{\tau} G_{T}$ extends to a spectral subalgebra of $C_{r}^{*}\left(\operatorname{Met}_{\tau} G_{T}\right)$. We show that a similar property holds for SMet ${ }_{\tau}^{(\omega)} G_{T}$ although it does not have the almost isometric property; that is, we show that $\phi$ extends to a subalgebra of $C_{r}^{*}\left(\operatorname{SMet}_{\tau}^{(\omega)} G_{T}\right)$ which has the same $K$-groups as $C_{r}^{*}\left(\operatorname{SMet}_{\tau}^{(\omega)} G_{T}\right)$, relying on a deep result on Oka's principle due to J.-B. Bost [B1].

We briefly recall the relevant constructions from [C7]. Let $E_{0}$ be a SMet ${ }_{\tau}^{(\omega)} G_{T^{-}}$ equivariant real vector bundle over $\operatorname{SMet}_{\tau}^{(\omega)} T$, endowed with a fiberwise metric. We do not assume that the metric is invariant under $\operatorname{SMet}_{\tau}^{(\omega)} G_{T}$. Let $E=E_{0} \otimes \mathbb{C}$ be its complexification with the Hermitian inner products $\left(\langle-,-\rangle_{E_{x}}\right)_{x \in \operatorname{SMet}_{\tau}^{(\omega)} G_{T}}$, and $\Gamma_{c}\left(\operatorname{SMet}_{\tau}^{(\omega)} G_{T} ; r^{*} E\right)$ be the space of compactly supported continuous maps $f: \operatorname{SMet}_{\tau}^{(\omega)} G_{T} \rightarrow E$ satisfying $f(\gamma) \in E_{r(\gamma)}$. Then $\Gamma_{c}\left(\operatorname{SMet}_{\tau}^{(\omega)} G_{T} ; r^{*} E\right)$ admits a 
$\operatorname{right} C_{c}\left(\operatorname{SMet}_{\tau}^{(\omega)} G_{T}\right)$-valued inner product

$$
\langle\xi, \eta\rangle_{C_{r}^{*}\left(\operatorname{SMet}_{\tau}^{(\omega)} G_{T}\right)}(\gamma)=\langle\xi(\gamma), \eta(\gamma)\rangle_{E_{r(\gamma)}} .
$$

This allows us to take the completion of $\Gamma_{c}\left(\operatorname{SMet}_{\tau}^{(\omega)} G_{T} ; r^{*} E\right)$ as a Hilbert $\mathrm{C}^{*}$ module over $C_{r}^{*}\left(\operatorname{SMet}_{\tau}^{(\omega)} G_{T}\right)$, denoted by $C_{r}^{*}\left(\operatorname{SMet}_{\tau}^{(\omega)} G_{T} ; E\right)$. The left convolution defines a closable (non- ${ }^{*}$-)homomorphism

$$
\lambda_{E}: C_{r}^{*}\left(\operatorname{SMet}_{\tau}^{(\omega)} G_{T}\right) \rightarrow \mathcal{L}\left(\Gamma_{c}\left(\operatorname{SMet}_{\tau}^{(\omega)} G_{T} ; r^{*} E\right)\right),
$$

whose domain is $C_{c}\left(\operatorname{SMet}_{\tau}^{(\omega)} G_{T}\right)$.

Lemma 16. Let $E_{0}$ be an $\operatorname{SMet}_{\tau}^{(\omega)}\left(G_{T}\right)$-equivariant real vector bundle, and $R$ be a real number. Suppose that $E_{0}$ is endowed with a metric satisfying

(11) $\langle\gamma(\xi), \gamma(\eta)\rangle_{r(\gamma)}=\delta(\gamma)^{2 R}\langle\xi, \eta\rangle_{s(\gamma)}, \quad\left(\gamma \in \operatorname{SMet}_{\tau}^{(\omega)}\left(G_{T}\right), \xi, \eta \in\left(E_{0}\right)_{s(\gamma)}\right)$.

Then, for any $f \in C_{c}\left(\operatorname{SMet}_{\tau}^{(\omega)} G_{T}\right)$, one has $\left\|\lambda_{E}(f)\right\|=\left\|\sigma_{-i R}^{(\omega)}(f)\right\|_{C_{r}^{*}\left(\operatorname{SMet}_{\tau}^{(\omega)} G_{T}\right)}$.

Proof. It is enough to find an operator $\tilde{S}$ on $C_{c}\left(\operatorname{SMet}_{\tau}^{(\omega)} G_{T} ; r^{*} E\right)$ satisfying the two conditions

$$
\langle\tilde{S}(\xi), \tilde{S}(\xi)\rangle_{C_{r}^{*}\left(\operatorname{SMet}_{\tau}^{(\omega)} G_{T}\right)}=\langle\xi, \xi\rangle_{C_{r}^{*}\left(\operatorname{SMet}_{\tau}^{(\omega)} G_{T}\right)}, \quad \tilde{S}\left(\lambda_{E}(f) \xi\right)=\tilde{S}(\xi) \sigma_{i R}\left(f^{*}\right) .
$$

We define $\tilde{S}$ by $\tilde{S}(\xi)(\gamma)=\delta(\gamma)^{-R} \gamma \cdot \overline{\xi\left(\gamma^{-1}\right)}$. Since $\xi\left(\gamma^{-1}\right) \in E_{r\left(\gamma^{-1}\right)}=E_{s(\gamma)}$ and $E$ has a distinguished real subbundle $E_{0}$, this is a well-defined transformation on $C_{c}\left(\operatorname{SMet}_{\tau}^{(\omega)} G_{T} ; r^{*} E\right)$.

On one hand, if $\gamma, \gamma^{\prime}, \gamma^{\prime \prime} \in \operatorname{SMet}_{\tau}^{(\omega)} G_{T}$ satisfies $\gamma^{-1}=\gamma^{\prime} \gamma^{\prime \prime}$, one has

$$
\delta(\gamma)^{-R} \gamma\left(\gamma^{\prime} \overline{\xi\left(\gamma^{\prime \prime}\right)}\right)=\delta\left(\gamma^{\prime}\right)^{R} \tilde{S}(\xi)\left(\left(\gamma^{\prime \prime}\right)^{-1}\right) .
$$

On the other hand, by definition of right convolution, one has

$$
\left(\eta \sigma_{i R}(f)\right)(\gamma)=\sum_{\bar{\gamma}^{\prime \prime} \bar{\gamma}^{\prime}=\gamma} \delta\left(\bar{\gamma}^{\prime}\right)^{R} \eta\left(\bar{\gamma}^{\prime \prime}\right) f\left(\bar{\gamma}^{\prime}\right) .
$$

Combining these, we obtain $\tilde{S}\left(\lambda_{E}(f) \xi\right)=\tilde{S}(\xi) \sigma_{i R}\left(f^{*}\right)$. Next, using the condition (11), one computes

$$
\langle\tilde{S}(\xi), \tilde{S}(\xi)\rangle_{C_{r}^{*}\left(\operatorname{SMet}_{\tau}^{(\omega)} G_{T}\right)}(\gamma)=\left\langle\xi\left(\gamma^{-1}\right), \xi\left(\gamma^{-1}\right)\right\rangle=\langle\xi, \xi\rangle_{C_{r}^{*}\left(\operatorname{SMet}_{\tau}^{(\omega)} G_{T}\right)}(\gamma) .
$$

This completes the proof.

Remark 17. Let $K=\mathrm{T} \operatorname{ker} p$ be the tangent bundle of the fibers for the fiber bundle $p$ : $\operatorname{SMet}_{\tau}^{(\omega)} T \rightarrow T$. Since $\mathrm{GL}_{k}(\mathbb{R}) / O_{k}(\mathbb{R})$ has a $\mathrm{GL}_{k}(\mathbb{R})$-invariant metric, 
$K$ is a $\operatorname{SMet}_{\tau}^{(\omega)} G_{T}$-equivariant real vector bundle with an invariant metric. We define $D_{0}^{\prime}=p^{*} \mathrm{~T} T \oplus K$, and $D_{0}=\bigoplus_{k=1}^{q^{\prime}}\left(D_{0}^{\prime}\right)^{\otimes k}$. The tautological metric on $p^{*} \mathrm{~T} T$ satisfies the assumption of Lemma 16 with $R=1$. It follows that $E_{0}^{\prime}$ is a direct sum of vector bundles satisfying this assumption with $R=0,1, \ldots, q^{\prime}$.

Let $E_{0}^{\prime}$ be the tangent bundle of $\operatorname{SMet}_{\tau}^{(\omega)} T$, endowed with a natural action of $\operatorname{SMet}_{\tau}^{(\omega)} G_{T}$. We fix a subbundle $N \subset E_{0}^{\prime}$ which satisfies $K \cap N=0$ and $K+N=E_{0}^{\prime}$. This allows us to define a metric on $E_{0}$ which is uniquely determined by the conditions $N \perp K, N \simeq p^{*} \mathrm{~T} T$. In other words, $D_{0}^{\prime}$ and $E_{0}^{\prime}$ coincide as metric bundles over $\operatorname{SMet}_{\tau}^{(\omega)} T$, only $\operatorname{SMet}_{\tau}^{(\omega)} G_{T}$ acts differently. As in the case of $D_{0}$, we put $E_{0}=\bigoplus_{k=1}^{q^{\prime}}\left(E_{0}^{\prime}\right)^{\otimes k}$.

Proposition 18. There is a Banach subalgebra $\mathcal{A}$ of $C_{r}^{*}\left(\operatorname{SMet}_{\tau}^{(\omega)} G_{T}\right)$ such that

1. $\mathcal{A}$ is contained in the domain of the closure of $\lambda_{E}$,

2. the inclusion $\mathcal{A} \rightarrow C_{r}^{*}\left(\operatorname{SMet}_{\tau}^{(\omega)} G_{T}\right)$ induces isomorphisms of K-groups,

3. the action $\sigma$ restricts to a strongly continuous action on $\mathcal{A}$.

Proof. We freely use the notation of Remark 17 in this proof.

First, put $\|f\|_{0}=\sup _{|R| \leq q^{\prime}}\left\|\sigma_{i R}(f)\right\|_{C_{r}^{*}\left(\operatorname{SMet}_{\tau}^{(\omega)} G_{T}\right)}$. By Lemma 16, $\lambda_{D}$ is continuous with respect to $\|\mid-\|_{0}$. Moreover, [B1, Theorem 1.1] implies that the closure $\mathcal{A}_{0}$ of $C_{c}\left(\operatorname{SMet}_{\tau}^{(\omega)} G_{T}\right)$ under $\|-\|_{0}$ has the same $K$-groups as $C_{r}^{*}\left(\operatorname{SMet}_{\tau}^{(\omega)} G_{T}\right)$. Moreover, by Lemma 16 and Remark 17, $\lambda_{D}$ defines a contractible representation of $\mathcal{A}_{0}$ on $C_{r}^{*}\left(\operatorname{SMet}_{\tau}^{(\omega)} G_{T} ; D\right)$.

Next, $K$ is a $\operatorname{SMet}_{\tau}^{(\omega)} G_{T}$-invariant subbundle of $E_{0}^{\prime}$. Thus, the action of $G_{T}$ on $E_{0}^{\prime}$ can be represented as a triangular block matrix with orthogonal matrices in the block diagonals, with respect to the decomposition $E_{0}^{\prime} \simeq K \oplus p^{*} \mathrm{TT}[\mathrm{C} 7$, Lemma 5.2]. This implies that there is a linear map $P$ from $C_{c}\left(\operatorname{SMet}_{\tau}^{(\omega)} G_{T}\right)$ to the space of linear transformations on $\Gamma_{c}\left(\operatorname{SMet}_{\tau}^{(\omega)} G_{T} ; E^{\prime}\right)$ such that $\lambda_{E^{\prime}}(f)=\lambda_{D^{\prime}}(f)+$ $P(f)$. As in the proof of [C7, Lemma 3.4], $\lambda_{E^{\prime}}(f)$ is conjugate to $\lambda_{D^{\prime}}(f)+\epsilon P(f)$ for any $\epsilon>0$. Then, we can find linear maps $P_{1}, \ldots, P_{q^{\prime}}$ from $C_{c}\left(\operatorname{SMet}_{\tau}^{(\omega)} G_{T}\right)$ to the space of linear transformations on $\Gamma_{c}\left(\operatorname{SMet}_{\tau}^{(\omega)} G_{T} ; E\right)$ such that $\lambda_{E}(f)$ is conjugate to $\lambda_{\epsilon}(f)=\lambda_{D}(f)+\sum_{k=1}^{q^{\prime}} \epsilon^{k} P_{k}(f)$ for any $\epsilon>0$.

Now, we can imitate the proof of [C7, Proposition 3.5]. We define $\mathcal{A}$ to be the intersection of $\mathcal{A}_{0}$ and the domain of the closure of $\lambda_{E}$. It remains to show that $\mathcal{A}$ is closed under holomorphic functional calculus in $\mathcal{A}_{0}$. For this, it is enough to show that, whenever $f \in \mathcal{A}$ satisfies $\|f\|_{0}<1$, the inverse of $1+f$ lies in $\mathcal{A}_{0}$ [C7, proof of Lemma 1.2]. Suppose that this assumption holds. Then $\left\|\lambda_{D}(f)\right\|$ is also smaller than 1 . Since the mapping $(0,1) \ni \epsilon \mapsto \lambda_{\epsilon}(f)$ is continuous and has limit $\lambda_{D}(f)$ at $\epsilon=0$, one has to have $\left\|\lambda_{\epsilon}(f)\right\|<1$ for a certain $\epsilon$. Now, the algebra $\mathcal{A}$ is equal to 
the closure of $C_{c}\left(\operatorname{SMet}_{\tau}^{(\omega)} G_{T}\right)$ with respect to the norm $\|f\|=\sup \left(\|f\|_{0},\left\|\lambda_{\epsilon}(f)\right\|\right)$ for this $\epsilon$. Thus, the inverse of $1+f$ exists in $\mathcal{A}$.

Finally, it remains to verify that $\sigma$ restricts to a strongly continuous action on $\mathcal{A}$. By definition of $\|-\| \|_{0}, \sigma$ restricts to a continuous action on $\mathcal{A}_{0}$. Similarly, the multiplier $\delta(\gamma)$ defines a strongly continuous 1-parameter automorphism group on $\mathcal{L}\left(C_{r}^{*}\left(\operatorname{SMet}_{\tau}^{(\omega)} G_{T} ; E\right)\right)$, which extends $\sigma$ via $\lambda_{E}$. Thus, we deduce that $\sigma$ is strongly continuous on $\mathcal{A}$. This completes the proof.

We are ready to apply the results of Section 3 to the transverse fundamental cocycle $\phi$. By [C7, Theorem 3.7], $\phi$ is a $q^{\prime}$-trace with respect to the norm $\|f\|$ for $\epsilon=1$ in the above proof. Thus, there exists a Fréchet subalgebra $\mathcal{A}_{0}^{\infty}$ of $\mathcal{A}$ which is closed under holomorphic functional calculus, and to which $\phi$ extends as a $q^{\prime}$-cyclic cocycle [C7, Section 2]. The symmetry of the seminorms used to define $\mathcal{A}_{0}^{\infty}$ implies that $\sigma$ acts strongly continuously on $\mathcal{A}_{0}^{\infty}$. We define $\mathcal{A}^{\infty}$ to be the subalgebra of $\mathcal{A}_{0}^{\infty}$ consisting of all smooth elements of $\sigma$. This is again stable under holomorphic functional calculus in $\mathcal{A}_{0}^{\infty}$. Since we ensured that the intermediate constructions between $C_{r}^{*}\left(\operatorname{SMet}_{\tau}^{(\omega)} G_{T}\right)$ and $\mathcal{A}^{\infty}$ have the same $K$-groups, we conclude that the inclusion $\mathcal{A}^{\infty} \rightarrow C_{r}^{*}\left(\operatorname{SMet}_{\tau}^{(\omega)} G_{T}\right)$ induces isomorphisms of $K$-groups.

\section{$\S 4.1$. Anabelian cocycles}

Definition 19 ([C7, Theorem 7.14; C8, Section 3.6. $\gamma$, Definition 18]). Let $A$ be a $\mathrm{C}^{*}$-algebra, $\mathcal{A}$ be its dense algebra, and $B$ be a unital subalgebra of the center $Z(\mathcal{M}(A))$ of the multiplier algebra of $A$. A cyclic $k$-cocycle $\psi$ on $B \mathcal{A}$ is said to be $B$-anabelian if the $(k+1)$-linear map on $\mathcal{A}$

$$
\psi_{g}\left(f^{0}, \ldots, f^{k}\right)=\psi\left(g f^{0}, f^{1}, \ldots, f^{k}\right)
$$

is a cyclic $k$-cocycle for any $g \in B$.

Lemma 20. Let $A, \mathcal{A}, B$ be as above, and let $\psi$ be a $k$-cocycle on $\mathcal{A}$. Suppose that $\psi$ extends to a cyclic cocycle on $(\mathcal{A}+B)^{k+1}$ and satisfies

$$
\psi\left(g, f^{1}, \ldots, f^{k}\right)=0
$$

for any $g \in B$. Then $\psi$ is B-anabelian.

Proof. The Hochschild cocycle property of $\psi_{g}$ follows from that of $\psi$ and from the fact that $g$ commutes with any element of $\mathcal{A}$. Hence it remains to show that $\psi_{g}$ satisfies the cyclicity condition.

The cyclicity of $\psi$ and (12) implies

$$
\psi\left(f^{j}, \ldots, f^{k}, g, f^{1}, \ldots, f^{j-1}\right)=0
$$


for any $1 \leq j \leq k$. The Hochschild cocycle condition implies

$$
\begin{aligned}
0= & b \psi\left(f^{0}, g, f^{1}, \ldots, f^{k}\right) \\
= & \psi\left(f^{0} g, f^{1}, \ldots, f^{k}\right)-\psi\left(f^{0}, g f^{1}, f^{2}, \ldots, f^{k}\right) \\
& +\sum_{j=2}^{k}(-1)^{j} \psi\left(f^{0}, g, f^{1}, \ldots, f^{j-1} f^{j}, \ldots, f^{k}\right)+(-1)^{k} \psi\left(f^{k} f^{0}, g, f^{1}, \ldots, f^{k-1}\right) .
\end{aligned}
$$

Combined with (13) and the cyclicity of $\psi$, this implies the cyclicity of $\psi_{g}$.

Proposition 21. Suppose that $(M ; F), T$, and $\omega$ satisfy the conditions $(\mathrm{PI})$ and (UE). Then the dual $\hat{\phi}$ on $\mathbb{R} \ltimes_{\sigma} C_{c}^{\infty}\left(\operatorname{SMet}_{\tau}^{(\omega)} G_{T}\right)$ of the transverse fundamental cocycle is $C^{\infty}\left(\operatorname{Det}_{\tau}^{+} T\right)^{G_{T}}$-anabelian.

Proof. By Lemma 20, it is enough to show the equality

$$
\hat{\phi}\left(g, f^{1}, \ldots, f^{q^{\prime}}\right)=0
$$

for any $G_{T}$-invariant function $g$ on $\operatorname{Det}_{\tau}^{+} T$. Recall the $\operatorname{decomposition}_{\operatorname{Det}_{\tau}^{+}}(T) \simeq$ $T \times \mathbb{R}_{>0}$ determined by the choice of $\omega$. Then we can write

$$
\psi\left(g, f^{1}, \ldots, f^{q^{\prime}}\right)=\int_{\mathbb{R}_{>0}} \frac{d t}{t} \int_{\operatorname{SMet}_{\tau}^{(\omega)}(T) \times\{t\}} g(p(x), t) d f^{1} \cdots d f^{q^{\prime}},
$$

where $p: \operatorname{Det}_{\tau}^{+}(T) \rightarrow T$ is the natural projection.

By the condition (UE), the function $g(y, t)$ on $T \times \mathbb{R}_{>0} \simeq \operatorname{Det}_{\tau}^{+}(T)$ is constant for each $t$. Hence

$$
\int_{\operatorname{SMet}_{\tau}^{(\omega)}(T)} g(p(x), t) d f^{1} \cdots d f^{q^{\prime}}=0
$$

which proves that the integral on the right hand side of (14) is trivial.

Theorem 2. Suppose that $(M ; F), T$, and $\omega$ satisfy the conditions (PI) and (UE). For each $x$ in $K_{q^{\prime}}\left(C_{r}^{*}\left(\operatorname{Met}_{\tau} G_{T}\right)\right)$, there is a $\theta$-invariant normal functional $\nu_{x}$ on the center of $\mathbb{R} \ltimes_{\sigma} W(M ; F)$ satisfying $\nu_{x}(1)=\langle\hat{\phi}, x\rangle$.

Proof. When $f \in C^{\infty}\left(\operatorname{Det}_{\tau}^{+}(T)\right)^{G_{T}}$, we define

$$
\nu_{x}(f)=\left\langle\hat{\phi}_{f}, x\right\rangle .
$$

The right hand side is normal as a functional defined on a weakly dense subspace of $L^{\infty}\left(\operatorname{Met}_{\tau}(T)\right)^{G_{T}}$. Moreover it satisfies $\nu_{x}(1)=\langle\hat{\phi}, x\rangle$. Hence it extends to a normal functional over $L^{\infty}\left(\operatorname{Met}_{\tau}(T)\right)^{G_{T}}$.

From $\left.\hat{\sigma}\right|_{\mathbb{R} \ltimes C_{r}^{*}\left(G_{T}\right)}=\theta$ and the $\hat{\sigma}$-invariance of the cocycle $\hat{\phi}$, we have

$$
\left\langle\theta_{t}^{*} \hat{\phi}, x\right\rangle=\left\langle\hat{\phi}, \hat{\sigma}_{t}(x)\right\rangle \text {. }
$$


Since $\hat{\sigma}_{t}(x)$ is a constant family in $K_{*}\left(C^{*} \operatorname{Met}_{\tau}\left(G_{T}\right)\right)$, one has $\left\langle\hat{\phi}, \hat{\sigma}_{t}(x)\right\rangle=\langle\hat{\phi}, x\rangle$. This proves the invariance of $\nu_{x}$ under $\theta$.

Corollary 22. With the same assumption as in Theorem 2, assume moreover that there exists an element $x \in K_{q^{\prime}}\left(C_{r}^{*}\left(\operatorname{Met}_{\tau} G_{T}\right)\right)$ such that $\langle\hat{\phi}, x\rangle \neq 0$. Then $W(M ; F)$ is a factor of type III.

Proof. The flow of weights of a semifinite factor is isomorphic to the translation of $\mathbb{R}$ on $L^{\infty}(\mathbb{R})$. Hence in that case there cannot be an invariant normal functional on $Z\left(\mathbb{R} \ltimes_{\sigma} W(M ; F)\right)[\mathrm{C} 7]$.

\section{$\S 5$. Period of flow and $K$-cycles}

In this section we apply the considerations of Section 3 to the situation of Theorem 2 and calculate the possible values of the pairing between the dual fundamental cocycle and the $K$-group when the von Neumann algebra $W(M ; F)$ is a factor of type $\mathrm{III}_{\lambda}$ for some $0<\lambda<1$. We will use Corollary 12 to relate the $K$-theory pairing of the dual of the fundamental cocycle with $K_{q^{\prime}}\left(C_{r}^{*}\left(\operatorname{Met}_{\tau}\left(G_{T}\right)\right)\right)$ to that of the fundamental cocycle with $K_{q^{\prime}}\left(C_{r}^{*}\left(\operatorname{SMet}_{\tau}^{(\omega)}\left(G_{T}^{(u)}\right)\right)\right)$.

In order to construct nontrivial elements in the $K$-group of groupoid algebras, we consider the group $K_{*}^{\mathrm{top}}\left(G_{X}\right)$ and the assembly map [BC], [C7], [C5]

$$
\mu: K_{*}^{\mathrm{top}}\left(G_{X}\right) \rightarrow K_{*}\left(C_{r}^{*}\left(G_{X}\right)\right)
$$

for the base spaces $X=\operatorname{SMet}_{\tau}^{(\omega)}(T)$ and $\operatorname{Met}_{\tau}(T)$.

A cycle $c$ in $K_{0}^{\text {top }}\left(G_{X}\right)$ is represented by a quadruple $(N, f, E, D)$, where $N$ is a manifold endowed with a map $f: N \rightarrow X$ and a proper action of $G_{X}$ with respect to $f$. Furthermore $E$ is a $\mathbb{Z}_{2}$-graded $G_{X}$-equivariant vector bundle on $N$, and $D=\left(D_{x}\right)_{x \in X}$ is a family of odd elliptic operators on the fibers of $f$ with coefficient $E$. Here, $D$ is assumed to satisfy the $G_{X}$-equivariance condition $\gamma D_{s \gamma} \gamma^{-1}=D_{r \gamma}$ for $\gamma \in G$. Given such data $c$, the $G$-index $\operatorname{Ind}_{G}\left(D_{*}\right)$ of the family $\left(D_{x}\right)_{x \in X}$ defines an element $\mu(c)$ in $K_{0}\left(C_{r}^{*}\left(G_{X}\right)\right)$.

Similarly, the pairs of a $G_{X}$-equivariant map $f: N \rightarrow X$ and equivariant longitudinal elliptic operators over $N \times \mathbb{R}$ define odd cycles which are elements of $K_{1}^{\text {top }}\left(G_{X}\right)$, and elements in $K_{1}\left(C_{r}^{*}\left(G_{X}\right)\right)$ via the assembly map.

Now, let us consider the case of the base space $X=\operatorname{SMet}_{\tau}^{(\omega)}(T)$. In the following we shall describe the natural map

$$
\Phi^{\mathrm{top}}: K_{0}^{\mathrm{top}}\left(G_{X}\right) \rightarrow K_{1}^{\mathrm{top}}\left(\operatorname{Det}_{\tau}^{+}\left(G_{X}\right)\right),
$$

which is compatible with the Connes-Thom isomorphism $\Phi$ from $K_{0}\left(C_{r}^{*}\left(G_{X}\right)\right)$ to $K_{1}\left(\mathbb{R} \ltimes_{\sigma^{(\omega)}} C_{r}^{*}\left(G_{X}\right)\right)$ via the assembly map $\mu$. 
Suppose that an element $x$ of $K^{\operatorname{top}}\left(G_{X}\right)$ is represented by $\left(N, f, E, D_{*}\right)$ of a $G_{X}$-equivariant map $f: N \rightarrow X$ and a family $\left(D_{x}\right)_{x \in X}$ of odd elliptic operators on a $G_{X}$-equivariant graded vector bundle $E$. Then $N \times \mathbb{R}$ admits an action of $G$ defined by

$$
\gamma(x, r)=\left(\gamma x, \delta^{(\omega)}(\gamma) t\right)
$$

The induced map

$$
\tilde{f}: N \times \mathbb{R} \rightarrow X \times \mathbb{R} \simeq \operatorname{Det}_{\tau}^{+} X \simeq \operatorname{Met}_{\tau}(T)
$$

is $G_{X}$-equivariant under this action.

Put $Y=X \times \mathbb{R}^{2}$ and let $\tilde{\mathcal{E}}$ be the $G_{X}$-equivariant vector bundle $\tilde{\mathcal{E}}_{(x, v)}=$ $\mathcal{E}_{x} \otimes \mathbb{C}^{2}$. For each $(x, v) \in X \times \mathbb{R}^{2}$, put $\tilde{D}_{(x, v)}=D_{x} \otimes 1+\epsilon \otimes D_{\mathbb{R}^{2}}$, where $\epsilon$ is the grading operator on $\mathcal{E}$ and $D_{\mathbb{R}^{2}}$ is the Dirac operator on $\mathbb{R}^{2}$. The latter defines a $G_{X}$-elliptic operator and the data $\left(Y, \tilde{f},\left(\tilde{D}_{(x, v) \in X \times \mathbb{R}^{2}}\right)\right)$ is a family of equivariant fiberwise elliptic operators parametrized by $Y=\operatorname{Det}_{\tau}^{+}(X) \times \mathbb{R}$. Let $\Phi^{\text {top }}(x)$ denote this element of $K_{1}^{\mathrm{top}}\left(\operatorname{Det}_{\tau}^{+}\left(G_{X}\right)\right)$.

Similarly, we can define $\Phi^{\text {top }}: K_{1}^{\text {top }}\left(G_{X}\right) \rightarrow K_{0}^{\text {top }}\left(G_{X}\right)$ by Bott periodicity.

Lemma 23. We have the equality

$$
\mu\left(\Phi^{\mathrm{top}}\left(N, f, E, D_{*}\right)\right)=\Phi\left(\mu\left(N, f, E, D_{*}\right)\right)
$$

in $K_{*+1}\left(\mathbb{R} \ltimes_{\sigma^{(\omega)}} C_{r}^{*}\left(G_{X}\right)\right)$ for any cycle $\left(N, f, E, D_{*}\right)$ of $K_{*}^{\text {top }}\left(G_{X}\right)$.

Proof. Let $\alpha^{(t)}$ denote the action $\alpha_{s}^{(t)}(x)=\sigma_{t s}^{(\omega)}(x)$ of $\mathbb{R}$ over $C_{r}^{*}\left(G_{X}\right)$, and $A=$ $\left(A_{t}\right)_{t \in[0,1]}$ be the continuous field of $\mathrm{C}^{*}$-algebras over $[0,1]$ whose fiber $A_{t}$ at $t \in$ $[0,1]$ is given by the crossed product $\mathbb{R} \ltimes_{\alpha^{(t)}} C_{r}^{*}\left(G_{X}\right)$. Then the evaluation map $e_{0}: A \rightarrow A_{0}$ at $t=0$ is a $K K$-isomorphism, and $e_{1}: A \rightarrow A_{1}$ at $t=1$ can be considered as a $K K$-morphism

$$
\Lambda: C_{0}(\mathbb{R}) \otimes C_{r}^{*}\left(G_{X}\right) \simeq A_{0} \rightarrow A_{1} .
$$

The Connes-Thom isomorphism $K_{*}\left(C_{r}^{*}\left(G_{X}\right)\right) \rightarrow K_{*+1}\left(\mathbb{R} \ltimes_{\sigma^{(\omega)}} C_{r}^{*}\left(G_{X}\right)\right)$ is given by the composition of the Bott periodicity isomorphism

$$
K_{*}\left(C_{r}^{*}\left(G_{X}\right)\right) \rightarrow K_{*+1}\left(C_{0}(\mathbb{R}) \otimes C_{r}^{*}\left(G_{X}\right)\right) \simeq K_{*+1}\left(A_{0}\right)
$$

and the map induced by $\Lambda$.

Put $Z=X \times \mathbb{R}^{2} \times[0,1]$ and consider the action of $G_{X}$ on $Z$ by

$$
\gamma \cdot(x, v, t)=\left(\gamma \cdot x,\left(t \log \left(\delta^{(\omega)}\right)+v_{1}, v_{2}\right), t\right) .
$$

Then the groupoid algebra $C_{r}^{*}\left(G_{X} \ltimes Z\right)$ is isomorphic to $A$ as a $C([0,1])$-algebra. Given a geometric cycle $z=\left(N, f, E, D_{*}\right)$ for $G_{X}$, let $\tilde{z}$ be the geometric cycle 
$\left(N \times \mathbb{R}^{2} \times[0,1], p_{1}^{*}\left(D_{*}\right)\right)$ over $G_{X} \ltimes Z$. Then $e_{0}(\mu(\tilde{z}))=\mu(z)$ and $e_{1}(\mu(\tilde{z}))=$ $\Phi(\mu(z))$, which proves the assertion.

Let $c=(N, f, E, D)$ be a cycle in $K_{*}^{\text {top }}\left(\operatorname{Det}_{\tau}^{+}\left(G_{X}\right)\right)$. We take the map $N \rightarrow \mathbb{R}$ given by the composition of $f$ and the projection $\operatorname{Det}_{\tau}^{+} X \rightarrow \mathbb{R}$ induced by $\omega$. Then the inverse image $N_{0}$ of $X \times\{0\}$ in $N$ is a submanifold of codimension 1 , and it has a $G_{X}^{(u)}$-equivariant map into $X$. Moreover, $E$ and $D$ restrict to $N_{0}$. The data $\iota^{*}(c)=\left(N_{0},\left.f\right|_{N_{0}},\left.E\right|_{N_{0}},\left.D\right|_{N_{0}}\right)$ defines an element of $K_{*}^{\text {top }}\left(G_{X}\right)$. This correspondence defines a well-defined map $\Psi^{\text {top }}: K_{*}^{\text {top }}\left(\operatorname{Det}_{\tau}^{+}\left(G_{X}\right)\right) \rightarrow K_{*}^{\text {top }}\left(G_{X}\right)$.

It can be easily seen that this map corresponds to the homomorphism $\Psi$ of (6) via $\mu$.

Lemma 24. We have the equality

$$
\mu\left(\Psi^{\mathrm{top}}(N, x)\right)=\Psi(\mu(N, x))
$$

in $K^{\mathrm{top}}\left(G_{X}^{(u)}\right)$ for any $(N, x) \in K^{\mathrm{top}}\left(\operatorname{Det}_{\tau}^{+}\left(G_{X}\right)\right)$.

Let $A$ be the $\mathrm{C}^{*}$-algebra $C_{r}^{*}\left(\operatorname{SMet}_{\tau}^{(\omega)} G_{T}\right)$. As a consequence of Lemmas 23 and 24 , we have the following commutative diagram:

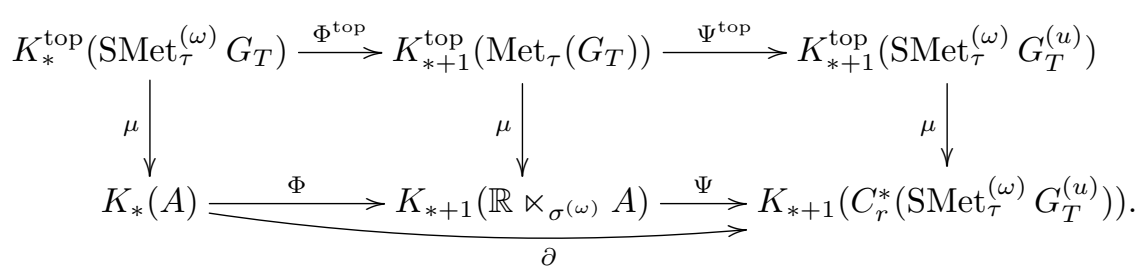

Theorem 3. Let $(M ; F)$ be a foliation, $T$ a transversal and $\omega$ a smooth density on $T$, satisfying the conditions (PI) and (UE). Suppose that the von Neumann algebra $W(M ; F)$ is of type $\mathrm{III}_{\lambda}$ for $0<\lambda<1$. Then

$$
\left\{\left\langle i_{D} \phi, \mu(x)\right\rangle \mid x \in K^{\mathrm{top}}\left(\operatorname{SMet}_{\tau}^{(\omega)}\left(G_{T}\right)\right)\right\} \subset \log (\lambda) \mathbb{Q} .
$$

Moreover,

$$
\log (\lambda) \mathbb{Z} \subset\left\{\left\langle i_{D} \phi, y\right\rangle \mid y \in K_{q+1}\left(C_{r}^{*}\left(\operatorname{SMet}_{\tau}^{(\omega)}\left(G_{T}\right)\right)\right)\right\} .
$$

Proof. By Lemma 5 and the assumption on the type of $W(M ; F)$, the spectrum of $\sigma^{(\omega)}$ agrees with $\log (\lambda) \mathbb{Z}$. By (UE), the action $\sigma^{(\omega)}$ is saturated.

By Proposition 18 and the following remark, we can find a $\sigma$-invariant Fréchet subalgebra $\mathcal{A}^{\infty}$ of $C_{r}^{*}\left(\operatorname{SMet}_{\tau}^{(\omega)} G_{T}\right)$ with the same $K$-groups, such that $\phi$ extends. Hence we may apply Corollary 12 , and obtain the equality

$$
\left\langle i_{D} \phi, y\right\rangle=\log (\lambda)\left\langle\phi \mid \frac{}{C_{c}^{\infty}\left(\operatorname{Met}_{\tau} G_{T}^{(u)}\right)}, \partial(y)\right\rangle
$$

for any $y \in K_{*}\left(C_{r}^{*}\left(G_{T}\right)\right)$. 
For any $(N, f, E, D) \in K_{q}^{\mathrm{top}}\left(G_{T}^{(u)}\right)$, we have

$$
\langle\phi, \mu(N, z)\rangle=\int_{N} \operatorname{Todd}(f) \operatorname{ch}\left(\sigma_{D}\right) \in \mathbb{Q},
$$

where $\operatorname{ch}\left(\sigma_{D}\right)$ denotes the Chern character of the symbol of $D$ in the compact support cohomology group $H_{c}^{*}(N, \mathbb{C})$, and Todd $(f)$ is the relative Todd class of $f$. This and (15) imply the first assertion.

Let $\left[G_{T}^{(u)}\right]^{*} \in K_{q}\left(C_{r}^{*}\left(G_{T}^{(u)}\right)\right)$ be the dual fundamental cocycle defined in [C5, Section 8]. It is represented by $\mu\left(D^{q}, x\right)$, where $D^{q} \rightarrow T$ is an immersion of the $q$-dimensional open disk and $x \in K^{q}\left(D^{q}\right)$ is the generator of $K^{q}\left(D^{q}\right) \simeq \mathbb{Z}$.

We show that $\left[G_{T}^{(u)}\right]^{*}$ is in the image of $K_{q+1}\left(C_{r}^{*}\left(G_{T}\right)\right)$, which is equivalent to

$$
(1-\hat{\sigma})\left(\Xi\left(\left[G_{T}^{(u)}\right]^{*}\right)\right)=0
$$

for the isomorphism $\Xi: K_{*}\left(C_{r}^{*}\left(G_{T}^{(u)}\right)\right) \rightarrow K_{*}\left(\mathbb{T} \ltimes_{\sigma^{(\omega)}} C_{r}^{*}\left(G_{T}\right)\right)$ induced by strong Morita equivalence.

Let $G_{T} \times_{\omega} \mathbb{Z}$ be the groupoid whose object set is $T \times \mathbb{Z}$, the arrow set is $G_{T} \times \mathbb{Z}$, and the structure maps are given by

$$
s(\gamma, n)=(s \gamma, n), \quad r(\gamma, n)=\left(r \gamma, n+\frac{\log \left(\delta^{(\omega)}(\gamma)\right.}{\log \lambda}\right) .
$$

Then $G_{T} \times_{\omega} \mathbb{Z}$ is strongly Morita equivalent to $G_{T}^{(u)}$ as a groupoid, and one has an isomorphism

$$
C_{r}^{*}\left(G_{T} \times \mathbb{Z}\right) \simeq \mathbb{T} \ltimes_{\sigma(\omega)} C_{r}^{*}\left(G_{T}\right)
$$

of algebras. Under this isomorphism the dual action $\hat{\sigma}$ on $\mathbb{T} \ltimes_{\sigma^{(\omega)}} C_{r}^{*}\left(G_{T}\right)$ corresponds to the action of $\mathbb{Z}$ on $G_{T} \times_{\omega} \mathbb{Z}$ given by $\alpha_{m}(\gamma, n)=(\gamma, n+m)$.

If $\Xi\left(\left[G_{T}^{(u)}\right]^{*}\right)$ is represented by an immersion $D^{q} \rightarrow T \times\{n\}$ for some $\iota: D^{q} \rightarrow T$ and $n \in \mathbb{Z}$, then $\alpha\left(\Xi\left(\left[G_{T}^{(u)}\right]^{*}\right)\right)$ is represented by $(\iota, n+1): D^{q} \rightarrow T \times\{n+1\}$. By replacing $D^{q}$ with a smaller disk if necessary, we may assume that the image of $\iota$ is contained in the domain of an element $\gamma \in G_{T}$ satisfying $\delta^{(\omega)}(\gamma)=\lambda$. Then $\gamma \circ \iota$ is an immersion of $D^{q}$ into $T$ and one has the equality

$$
(\gamma \circ \iota, 1)_{*}\left[D^{q}\right]^{*}=((\gamma, 0) \circ(\iota, 0))_{*}\left[D^{q}\right]^{*}=\gamma\left(\iota_{*}\left(\left[D^{q}\right]^{*}\right)\right) \gamma^{*}=\iota_{*}\left[D^{q}\right]^{*}
$$

in $K_{q}\left(C_{r}^{*}\left(G_{T} \times \omega \mathbb{Z}\right)\right)$. By the condition (UE), there is an element $\gamma_{1}$ in $G_{T}^{(u)}$ whose domain contains the image of $\gamma \circ \iota$ and codomain lies in the same connected component as the image of $\iota$. With this $\gamma_{1}$ one has

$$
(\gamma \circ \iota, 1)_{*}\left[D^{q}\right]^{*}=\gamma_{1}((\gamma \circ \iota, 1))_{*}\left[D^{q}\right]^{*} \gamma_{1}^{*}=(\iota, 1)_{*}\left[D^{q}\right]=\alpha\left((\iota, 0)_{*}\left[D^{q}\right]^{*}\right) .
$$


Combining (17) and (18), we obtain $\alpha\left(\iota_{*}\left[D^{q}\right]^{*}\right)=\iota_{*}\left[D^{q}\right]^{*}$, which proves (16). From $\left\langle\phi,\left[G_{T}^{(u)}\right]^{*}\right\rangle=1,(15)$, and Corollary 12 , we obtain

$$
\log (\lambda) \in\left\langle i_{D} \phi, K_{q+1}\left(C_{r}^{*}\left(\operatorname{SMet}_{\tau}^{(\omega)}\left(G_{T}\right)\right)\right)\right\rangle .
$$

This proves the latter half of the assertion.

\section{$\S 5.1$. The 1-form corresponding to the dual of fundamental cocycle}

In this last section, we consider the case where $M$ is compact and there is a covering $\pi: M^{\prime} \rightarrow M$ and a holonomy invariant transverse density $\omega$ of $\left(M^{\prime}, \pi^{*} F\right)$ such that $\omega$ is projectively invariant under the deck transformation group of $M^{\prime}$. Under this assumption, if we take a transversal $T$ which admits a section $f: T \rightarrow M^{\prime}$, the pullback of $\omega$ on $T$ by $f$ defines a projectively invariant transverse density for $F$.

The Radon-Nikodym cocycle of the deck transformation group with respect to the density $\omega$ defines an $\mathbb{R}_{+}$-valued group 1-cocycle. By taking the natural logarithm and considering the double complex of $\Omega^{*}\left(M^{\prime}\right)$-valued $\Gamma$-cochains, this group cocycle can be regarded as a class $\left[\log \delta^{(\omega)}\right]$ in $H^{1}(M ; \mathbb{R})$.

We can also define $\left[\log \delta^{(\omega)}\right]$ by a Čech 1-cocycle in the following way. Let $\left(U_{i}\right)_{i \in I}$ be a covering of $M$ by foliation charts admitting sections $f_{i}: U_{i} \rightarrow M^{\prime}$. Then for each pair $(i, j)$ of indices there exists a unique element $g_{i j}$ of the deck transformation group. The scalar $c_{i j}$ characterized by $c_{i j} \omega=g_{i j}^{*} \omega$ satisfies the Cech 1-cocycle identity with respect to the group law of $\mathbb{R}_{+}^{\times}$. The $\mathbb{R}$-valued Cech 1-cocycle $\left(\log \left(c_{i j}\right)\right)_{i j}$ for $\left(U_{i}\right)_{i \in I}$ defines the desired class $\left[\log \delta^{(\omega)}\right]$.

For each open set $U$ of $M$, let $\mathcal{A}_{U}$ be the convolution algebra of compactly supported smooth functions over the full holonomy groupoid of $\left.F\right|_{U}$. On the one hand, any family $\left(D_{l}\right)_{l \in M / F}$ of leafwise elliptic operators defines an element $\operatorname{Ind}(D)$ of the $K_{0}$-group of $\mathcal{A}_{M}$. On the other hand, by strong Morita equivalence, there is a natural isomorphism $\mathrm{HC}^{n}\left(C_{c}^{\infty}\left(G_{T}\right)\right)$ and $\operatorname{HC}^{n}\left(\mathcal{A}_{M}\right)$ for each $n$.

Next, recall that there is a map of $\mathbb{Z}_{2}$-graded vector spaces [C8, Section 3.7. $\gamma$ ]

$$
\Lambda: \operatorname{HP}^{*}\left(\mathcal{A}_{M}\right) \rightarrow\left(\bigoplus_{k \in q+2 \mathbb{Z}} H^{k}(M)\right) \oplus\left(\bigoplus_{k \in 1+q+2 \mathbb{Z}} H^{k}(M)\right)
$$

which satisfies

$$
\langle\operatorname{Ind}(D), \psi\rangle=\int_{M} \operatorname{Todd}(\tau \otimes \mathbb{C})^{-1} \Phi_{H}(\operatorname{ch}([\sigma(D)])) \Lambda(\psi),
$$

where $\Phi_{H}$ is the Thom isomorphism $H_{c}^{*}\left(F^{*}\right) \rightarrow H_{c}^{*-p}(M)$ in cohomology. Let us recall the construction of $\Lambda$.

We take an open covering $\mathcal{U}=\left(U_{i}\right)_{i \in I}$ of $M$. For each open set $U \subset M$ and $k \in \mathbb{N}$, put

$$
\Omega_{F}^{k}(U)=\left(\mathcal{A}_{U}^{\otimes k} \oplus \mathcal{A}_{U}^{\otimes k+1}\right)^{\prime} .
$$


Next, for such $U$ and $k$, let $\Omega_{k}^{\tau}(U)$ denote the space of $\left.F\right|_{U}$-holonomy invariant transverse $k$-currents on $U$. By choosing a transverse bundle $H \subset \mathrm{T} M$, one can define a map of complexes

$$
\Omega_{k}^{\tau}(U) \rightarrow \Omega_{F}^{k}(U)
$$

for any $k$, natural in $U$, and which does not depend on the choice of $H$ when one passes to cohomology.

Note that when $U_{1} \subset U_{2}$ are open sets of $M$, we have natural maps $\Omega_{F}^{k}\left(U_{2}\right) \rightarrow$ $\Omega_{F}^{k}\left(U_{1}\right)$ and $\Omega_{k}^{\tau}\left(U_{2}\right) \rightarrow \Omega_{k}^{\tau}\left(U_{1}\right)$.

We then consider the following triple complexes $\Gamma_{\mathcal{U}}^{a, b, c}$ and $\Gamma_{\mathcal{U}}^{a, b, c}$. The first one is given by

$$
\Gamma_{\mathcal{U}}^{a, b, c}=\prod_{i_{1}<\cdots<i_{c}} \Omega_{F}^{b-a}\left(U_{i_{1}} \cap \cdots \cap U_{i_{c}}\right),
$$

together with the differential $B$ (which increases the index $a$ ), the Connes-Reinhart operator $b$ (which increases the index $b$ ), and the Cech coboundary map (which increases the index $c$ ). Similarly, the second triple complex is given by

$$
\Gamma_{\mathcal{U}}^{\prime a, b, c}=\prod_{i_{1}<\cdots<i_{c}} \Omega_{b-a}^{\tau}\left(U_{i_{1}} \cap \cdots \cap U_{i_{c}}\right) .
$$

with the de Rham differential $d$ (which increases the index $a$ ), the zero differential (increasing $b$ ), and the Cech coboundary map (increasing $c$ ). Then the map (20) induces a map of complexes

$$
\Lambda_{0}: \Gamma_{\mathcal{U}}^{a, b, c} \rightarrow \Gamma_{\mathcal{U}}^{a, b, c}
$$

which is a quasi-isomorphism when each $U_{i}$ is contained in a foliation chart.

When $U$ is a foliation chart of $F$ homeomorphic to $T \times V$ for $T \simeq \mathbb{R}^{q}$ and $V \simeq \mathbb{R}^{p}$, we have the isomorphism $\mathcal{A}_{U} \simeq C_{c}^{\infty}(T) \hat{\otimes} \mathcal{K}^{\infty}$, which implies

$$
\operatorname{HP}^{n}\left(\mathcal{A}_{U}\right) \simeq \begin{cases}\mathbb{C} & (q \equiv n \bmod 2), \\ 0 & (q \neq \equiv \bmod 2) .\end{cases}
$$

On the other hand we also have $\Omega_{k}^{\tau}(U)=\Omega_{k}(T)$ for such $U$. It follows that the cohomology of the complex $\Omega_{*}^{\tau}(U)$ is trivial except in degree $q$, and that $H_{q}\left(\Omega_{*}^{\tau}(U)\right)=\mathbb{C}$. When $\left(U_{i}\right)_{i \in I}$ is a good cover of $M$ by foliation charts, the cohomology of the triple complex $\Gamma_{\mathcal{U}}^{\prime a, b, c}$ is equal to the Cech cohomology of the orientation sheaf of $\tau$ (up to shift in degree), which is isomorphic to the usual cohomology group of $M$.

Hence the cohomology of the triple complex $\Gamma_{\mathcal{U}}^{a, b, c}$ is equal to the right hand side of (19). The restriction homomorphism $\Omega_{F}^{b-a}(M) \rightarrow \prod_{i \in I} \Omega_{F}^{b-a}\left(U_{i}\right)$ gives the required map $\Lambda$. 
Although the manifolds $\operatorname{Det}_{\tau}^{+}(M), \operatorname{Met}_{\tau}(M)$ are not compact, the above construction of $\Lambda$ still makes sense for the foliations induced by $F$ on these manifolds. For example, when $U$ is a foliation chart of $(M ; F)$, its inverse image in $\operatorname{Det}_{\tau}^{+}(M)$ becomes a foliation chart of the canonical lift $\tilde{F}$ of $F$. Thus $\left(\operatorname{Det}_{\tau}^{+}(M), \tilde{F}\right)$ admits a finite covering by foliation charts, which allows us to define the map $\Lambda: C_{c}^{\infty}\left(\operatorname{Det}_{\tau}^{+}(G)\right) \rightarrow H^{*}\left(\operatorname{Det}_{\tau}^{+}(M)\right)$ in the same way as in the compact case.

This correspondence $\Lambda$ has two important special cases. The first case concerns a holonomy invariant transverse measure (if any) of $(M ; F)$. In that case, the associated trace, which is a 0 -cyclic cocycle on $C_{c}(M ; F)$, is mapped to the Poincaré dual $q$-form of the Ruelle-Sullivan $p$-current. The second is that the transverse fundamental class is mapped to the class of the unit in $H^{0}(M)$.

Here, we are interested in the image of the $(q+1)$-cyclic cocycle $i_{D} \phi$ when $(M ; F)$ admits a projectively invariant transverse measure. From the consideration of parity, it follows that $\Lambda\left(i_{D} \phi\right)$ is a class of odd degree. The following theorem identifies this cohomology class.

Theorem 4. Suppose that there is a covering $\pi: M^{\prime} \rightarrow M$ and a holonomy invariant transverse measure $\omega$ of $\left(M^{\prime}, \pi^{*} F\right)$ which is projectively invariant under the deck transformations of $\pi$. Let $\phi$ be a transverse fundamental cocycle on $C_{c}^{\infty}\left(G_{T}\right)$, and $D$ be the derivation of the modular automorphism group associated with $\omega$. Then $\Lambda\left(i_{D} \phi\right)$ coincides with $\left[\log \delta^{(\omega)}\right]$ in $H^{1}(M)$.

In order to prove the above we take a well-behaved open sets of $M$ with respect to the given foliation.

Definition 25. An open set $U$ in $M$ is said to be $F$-straight when we have a complete transversal $T$ in $U$ with trivial holonomy for $\left.F\right|_{U}$.

Remark 26. Since any foliation chart is $F$-straight, $M$ admits a covering by $F$ straight open sets.

Let $U$ be an $F$-straight open set of $M$. Let $\tilde{U}$ be the preimage of $U$ under the projection map $\tilde{\pi}: \operatorname{Det}_{\tau}^{+}(M) \rightarrow M$. Then $\tilde{U}$ is a $\tilde{\pi}^{*} F$-straight open subset of $\operatorname{Det}_{\tau}^{+}(M)$. Let $f$ be a section of the covering map. Then the pullback of $\omega$ by $f$ determines a trivialization $\tilde{U} \simeq \mathbb{R} \times U$. We let $t$ denote the coordinate on the first component of the right hand side. Let $f^{0}, f^{1}$ be two sections $U \rightarrow M^{\prime}$, and $c_{f^{0}} / f^{1}$ be the scalar such that $c_{f^{0} / f^{1}} \omega=g^{*} \omega$ where $g$ is the deck transformation satisfying $g \circ f^{0}=f^{1}$. Then the $t$-coordinates determined respectively by $f^{0}$ and $f^{1}$ differ by translation by the constant $\log \left(c_{f^{0}} / f^{1}\right)$.

Proof of Theorem 4. We choose a covering $\left(U_{i}\right)_{i \in I}$ of $M$ by $F$-straight open sets, and let $\left(\tilde{U}_{i}\right)_{i \in I}$ be the corresponding covering of $\operatorname{Det}_{\tau}^{+}(M)$ by the inverse images 
of the $U_{i}$. For each $i$, we choose a section $f^{i}: U_{i} \rightarrow M^{\prime}$ and let $t_{i}$ denote the corresponding $t$-coordinate on $\tilde{U}_{i}$. The coordinate transforms between the $\tilde{U}_{i}$ are given by the ones for $U_{i}$, and the ones between the $t_{i}$-coordinates induced by the holonomy transformation. We see that the 1 -forms $d t_{i}$ on the sets $\tilde{U}_{i}$ are invariant under these coordinate transforms. Hence we obtain a global 1-form on $\operatorname{Det}_{\tau}^{+}(M)$, denoted by $d t$.

Since the projection map $\operatorname{Det}_{\tau}^{+}(M) \rightarrow M$ has the contractible fiber $\mathbb{R}_{+}^{\times}$, it induces an isomorphism of cohomology. It is easy to see that the Cech 1-cocycle corresponding to $d t$ is precisely the one used to define $\left[\log \delta^{(\omega)}\right]$. We claim that $d t$ is the image of $\hat{\phi}$ under $\Lambda: \operatorname{HP}^{*}\left(C_{c}^{\infty}\left(\operatorname{Det}_{\tau}^{+}(G)\right)\right) \rightarrow H^{*+q+1}\left(\operatorname{Det}_{\tau}^{+}(M)\right)$.

First, the restriction of $\hat{\phi}$ to $C_{c}^{\infty}\left(\tilde{U}_{i} ; \tilde{\pi}^{*} F\right)$ corresponds to the holonomy invariant transverse $q$-current on $\tilde{U}_{i}$ defined by

$$
\int_{\mathbb{R}} d t_{i} \int_{\{t\} \times T_{i}} f^{0} d f^{1} \cdots d f^{q} .
$$

Integration by parts shows that this current is the boundary of the invariant transverse $(q+1)$-current

$$
\psi_{i}\left(f^{0} d f^{1} \cdots d f^{q+1}\right)=\int_{\tilde{U}_{i}} t_{i} f^{0} d f^{1} \cdots d f^{q+1}
$$

The Čech coboundary of $\left(\psi_{i}\right)_{i \in I}$ is given by $\left(\left(t_{i}-t_{j}\right) \tilde{\phi}_{i j}\right)_{i, j \in I}$, where $\tilde{\phi}_{i j}$ is the transverse fundamental current for $\tilde{\pi}^{*} F$. This shows that $\Lambda(\hat{\phi})$ is indeed equal to the class of $d t$.

By Lemma 23, $i_{D} \phi$ and $\hat{\phi}$ induce the same map on geometric $K$-groups. From this it already follows that $\Lambda\left(i_{D} \psi\right)$ and $\left[\log \delta^{(\omega)}\right]$ pair the same way against homology classes of the form $\operatorname{Todd}(\tau \otimes \mathbb{C})^{-1} \operatorname{ch}\left(\sigma_{D}\right) \cap[M]$ for a longitudinal elliptic operator $D$. To see that they agree as elements of $H^{*}(M)$, we may use the fact that the construction of $\left.\hat{\phi}\right|_{C_{c}^{\infty}\left(\tilde{U} ; \tilde{\pi}^{*} F\right)}$ and the natural isomorphisms $\operatorname{HP}^{*}\left(C_{c}^{\infty}\left(\tilde{U} ; \tilde{\pi}^{*} F\right)\right) \rightarrow$ $\operatorname{HP}^{*+1}\left(C_{c}^{\infty}(U ; F)\right)$ for the open sets $U$ of $M$ are compatible with the localization maps used in the definition of $\Lambda$.

Remark 27. The setting of this section was already considered by H. Moriyoshi $[\mathrm{M}]$, who raised the question about the relationship between the structure of $W(M ; F)$ and a certain geometrically defined subset of $\mathbb{R}$, called the $K$-set of $(M ; F)$.

Let $H_{1}^{F}$ be the intersection of $H_{1}(M ; \mathbb{Z})$ and the joint kernel of all closed $F$-basic 1-forms. Intuitively, $H_{1}^{F}$ can be thought of as the span of the 1-cycles in the leaves of $F$. Then the $K$-set of $(M ; F)$ is defined as the values which $\left[\log \delta^{(\omega)}\right]$ takes on $H_{1}^{F}$. 
Suppose that the von Neumann algebra $W(M ; F)$ is of type $\mathrm{III}_{\lambda}$. Theorem 4 , combined with Theorem 3 and the compatibility of $\Lambda$ with $\Phi_{K}$, shows that the pairing of $\left[\log \delta^{(\omega)}\right]$ with the twisted Chern character of the cycles in $K_{*}^{\text {top }}(M ; F)$ is contained in $\mathbb{Q} \log (\lambda)$. If the Baum-Connes conjecture for $(M ; F)$ holds, the number $\log (\lambda)$ itself can be realized by some cycle. The set $\left\langle i_{D}, \phi, K_{*}^{\text {top }}(M ; F)\right\rangle$ will contain the $K$-set if the twisted Chern character of an element $y \in K_{*}^{\text {top }}(M ; F)$ satisfying $\partial(\mu(y))=\left[G_{T}^{(u)}\right]^{*}$ (see the proof of Theorem 3 ) lies in $H_{1}^{F}$, which happens in many examples.

\section{Acknowledgements}

This paper was part of the author's master thesis submitted to the University of Tokyo. He would like to thank Y. Kawahigashi for his continuing support throughout the research. He is also grateful to G. Skandalis, C. Oikonomides, H. Moriyoshi, T. Katsura, and T. Tsuboî for many fruitful exchanges. Last but not least, he thanks the referee for his valuable comments which were crucial to fix many ambiguities in the original manuscript.

\section{References}

[BC] P. Baum and A. Connes, Geometric $K$-theory for Lie groups and foliations, Enseign. Math. (2) 46 (2000), 3-42. Zbl 0985.46042 MR 1769535

[B1] J.-B. Bost, Principe d'Oka, $k$-théorie et systèmes dynamiques non commutatifs, Invent. Math. 101 (1990), 261-333. Zbl 0719.46038 MR 1062964

[B2] R. Bowen, Anosov foliations are hyperfinite, Ann. of Math. (2) 106 (1977), 549-565. Zbl 0374.58008 MR 0461584

[C1] A. Connes, Une classification des facteurs de type III, C. R. Acad. Sci. Paris Sér. A-B 275 (1972), A523-A525. Zbl 0243.46064 MR 0303306

[C2] Sur la théorie non commutative de l'intégration, in Algèbres d'opérateurs (Les Plans-sur-Bex, 1978), Lecture Notes in Math. 725, Springer, 1979, 19-143. Zbl 0412.46053 MR 0548112

[C3] An analogue of the Thom isomorphism for crossed products of a $C^{*}$-algebra by an action of R, Adv. Math. 39 (1981), 31-55. Zbl 0461.46043 MR 0605351

[C4] Feuilletages et algèbres d'opérateurs, in Bourbaki Seminar, Vol. 1979/80, Lecture Notes in Math. 842, Springer, 1981, 139-155. Zbl 0522.46043 MR 0636521

[C5] A survey of foliations and operator algebras, in Operator algebras and applications, Part I (Kingston, Ont., 1980), Amer. Math. Soc., Providence, RI, 1982, 521-628. Zbl 0531.57023 MR 0679730

[C6] , Noncommutative differential geometry, Inst. Hautes Études Sci. Publ. Math. 62 (1985), 257-360. Zbl 0592.46056 MR 0823176

[C7] Cyclic cohomology and the transverse fundamental class of a foliation, in Geometric methods in operator algebras (Kyoto, 1983), Pitman Res. Notes Math. Ser. 123, Longman, 1986, 52-144. Zbl 0647.46054 MR 0866491

[C8] - Noncommutative geometry, Academic Press, San Diego, CA, 1994. Zbl 0818.46076 MR 1303779 
[C9] J. Cuntz, Cyclic theory, bivariant $K$-theory and the bivariant Chern-Connes character, in Cyclic homology in non-commutative geometry, Encyclopaedia Math. Sci. 121, Springer, 2004, 1-72. MR 2052771

[ENN] G. A. Elliott, T. Natsume, and R. Nest, Cyclic cohomology for one-parameter smooth crossed products, Acta Math. 160 (1988), 285-305. Zbl 0655.46054 MR 0945014

[GHL] W. M. Goldman, M. W. Hirsch, and G. Levitt, Invariant measures for affine foliations, Proc. Amer. Math. Soc. 86 (1982), 511-518. Zbl 0534.57014 MR 0671227

[HK] S. Hurder and A. Katok, Secondary classes and transverse measure theory of a foliation, Bull. Amer. Math. Soc. (N.S.) 11 (1984), 347-350. Zbl 0593.58015 MR 0752795

[M] H. Moriyoshi, A secondary invariant of foliated spaces and type $\mathrm{III}_{\lambda}$ von Neumann algebras, in From geometry to quantum mechanics, Progr. Math. 252, Birkhäuser Boston, 2007, 277-286. Zbl 1129.57033 MR 2285046

[N] R. Nest, Cyclic cohomology of crossed products with Z, J. Funct. Anal. 80 (1988), 235283. Zbl 0658.46054 MR 0961899

[PV] M. Pimsner and D. Voiculescu, Exact sequences for $K$-groups and Ext-groups of certain cross-product $C^{*}$-algebras, J. Operator Theory 4 (1980), 93-118. Z Zbl 0474.46059 MR 0587369

[P] J. F. Plante, Anosov flows, transversely affine foliations, and a conjecture of Verjovsky, J. London Math. Soc. (2) 23 (1981), 359-362. Z Zbl 0465.58020 MR 0609116

[Y] M. Yamashita, Connes-Landi deformation of spectral triples, Lett. Math. Phys. 94 (2010), 263-291; arXiv:1006.4420 [math.OA]. Zbl 1207.58010 MR 2738561 